\title{
Three dimensional structure of the unsteady wake of an axisymmetric body
}

Cite as: Phys. Fluids 31, 025113 (2019); https://doi.org/10.1063/1.5078379

Submitted: 24 October 2018 . Accepted: 30 January 2019 . Published Online: 19 February 2019

Giancarlo Pavia (D), Max Varney, Martin Passmore (D), and Mathew Almond

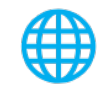

\section{ARTICLES YOU MAY BE INTERESTED IN}

On the development of low frequency structures in near and far laminar wakes

Physics of Fluids 31, 023604 (2019); https://doi.org/10.1063/1.5081050

Two-dimensionalization of a three-dimensional bluff body wake

Physics of Fluids 31, 017104 (2019); https://doi.org/10.1063/1.5066422

Influence of bed proximity on the three-dimensional characteristics of the wake of a sharpedged bluff body

Physics of Fluids 31, 025116 (2019); https://doi.org/10.1063/1.5085666

\section{Seilight Highlights of the best new research} in the physical sciences 


\title{
Three dimensional structure of the unsteady wake of an axisymmetric body
}

\author{
Cite as: Phys. Fluids 31, 025113 (2019); doi: 10.1063/1.5078379 \\ Submitted: 24 October 2018 - Accepted: 30 January 2019 • \\ Published Online: 19 February 2019
}

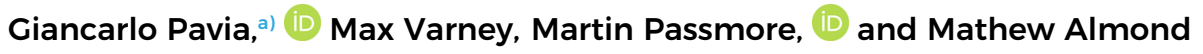

\author{
AFFILIATIONS \\ Stewart Miller Building, Loughborough University, Loughborough, Leicestershire LE11 3TU, United Kingdom
}

a) G.Pavia@lboro.ac.uk

\begin{abstract}
The near-wake of an axisymmetric body has been investigated using base pressure tappings and large scale Tomographic Particle Image Velocimetry (TPIV) at a Reynolds number of $\mathrm{Re}_{\mathrm{D}}=3.2 \times 10^{5}$, based upon model diameter. Insights into the near-wake dynamics are provided by the application of Proper Orthogonal Decomposition (POD) to the pressure and the TPIV datasets. The first two POD modes show that the axisymmetric topology seen in the time averaged field is the result of the combination of different reflectional symmetry preserving states, each one featuring a hairpin vortex surrounded by an annular structure developing in proximity to the wake closure. The "head" and the "tails" of each hairpin vortex appear to be dynamically linked, as also proven by the existence of a second pair of modes, visible only in the TPIV dataset, featuring a twisted two-lobe structure. The analysis of the temporal evolution of the radial position of the centre of pressure over the model base reveals the existence of two different low-drag scenarios, characterised by the restoration of the axial symmetry or the selection of a single plane of reflectional symmetry. The first state is reported to become the only admissible low-drag configuration when the short-time wake dynamics are removed from the unsteady pressure signal.
\end{abstract}

Published under license by AIP Publishing. https://doi.org/1 0.1063/1.5078379

\section{INTRODUCTION}

Flow around bluff bodies is of interest in many applications and, in particular, in the transport industry where the flow field is often characterised by large areas of separated flow. Such regions are usually the dominant contribution to the vehicle resistance, and thereby the fuel consumption, and can also be a significant source of noise for the vehicle occupants.

In the laminar regime, the flow past a body of revolution with a blunt base is dominated by a global instability with an azimuthal wave number $m= \pm 1$. The wake behind such geometries shows different flow patterns depending on the Reynolds number $\left(\mathrm{Re}_{\mathrm{D}}\right)$ and the length-to-diameter ratio (L/D). ${ }^{6}$ For example, Bury and Jardin ${ }^{7}$ showed that for a body with L/D $=7$, a uniform toroidal structure exists at the base of the body for Reynolds numbers around 100. As the Reynolds number increases, the torus expands in the radial direction and stretches downstream, as a result of the concomitant action of shear and suction forces. For Reynolds numbers around 450, the torus tends to move away from the body axis of symmetry. The shear stresses acting at the periphery of the torus are no longer uniform, and the torus loses its axi-symmetry, preserving only a single plane of symmetry aligned with the streamwise direction. This reflectional symmetry steady state is characterised by a "double-threaded" wake topology, consisting of two primary vorticity lobes located on each side of the reflectional symmetry plane. As the Reynolds number is further increased, the two lobes stretch in the streamwise direction and move away from the body's axis of revolution. Eventually, the structure can no longer sustain the shear stress and is partially torn (at $\mathrm{Re}_{\mathrm{D}} \approx 590$ ). In these conditions, unsteady hairpin vortices are shed in the wake at a Strouhal number $\left(\mathrm{St}_{\mathrm{D}}=f \cdot \mathrm{D} / \mathrm{V}_{\infty}\right.$, with $f$ denoting the frequency and $\mathrm{V}_{\infty}$ denoting the value of the free stream velocity) of approximately 0.12 . The reflectional symmetry, however, is preserved and is consequently generally described as a reflectional symmetry preserving (RSP) state. At Reynolds numbers above 690, 
a second peak at a Strouhal number of $\approx 0.02$ occurs. This peak has been shown to be associated with vorticity bursts that yield a waving motion of the hairpin structures along the reflectional symmetry plane. The reflectional symmetry preserving state persists up to Reynolds numbers of about 900, when the wake shifts toward a chaotic regime. The reflectional symmetry plane still exists but now only for short time intervals, and it is randomly reoriented on a very long time scale.

Similar regimes have been observed for the wakes developing downstream of other axisymmetric bodies such as spheres and circular flat disks, ${ }^{24}$ although with some differences. Fabre et al. $^{13}$ reported the existence of two additional vortex shedding modes for the wake behind a flat disk. The first, dubbed reflectional symmetry breaking (RSB) mode, is characterised by the twisting of the main vortical structures around the body's axis of revolution. It originates from the steady symmetric state following a Hopf bifurcation at $R e_{D}$ $=123$, and it was shown to be unstable using linear stability analysis. A second mode was found at $R e_{\mathrm{D}} \approx 140$, and it was described as a standing wave mode. Similar modes are reported by Auguste et al. ${ }^{3}$ for the wake behind a disk with $\mathrm{L} / \mathrm{D}=1 / 3$.

The effects of the bifurcating oscillatory modes seen in the laminar regime persist at high Reynolds numbers, resulting in the shedding of large coherent structures. In the case of a disk, the near-wake dynamics are dominated by the action of antisymmetric modes with $m= \pm 1$ and a constant shedding frequency. ${ }^{14}$ In addition, for Reynolds numbers above 1000, hairpin vortices are shed from the disk, but the angle of the shedding changes irregularly from cycle to cycle. ${ }^{30}$ For the same geometry, a second mode with a wave number $m=0$ was observed by Berger et al. ${ }^{5}$ Known as the "pumping mode," this results from the alternate expansion and shrinkage of the recirculating zone, with a characteristic frequency of $\mathrm{St}_{\mathrm{D}}=0.05$.

Rigas et al., ${ }^{36}$ applying proper orthogonal decomposition (POD) to a set of pressure data recorded over the base of an axisymmetric bluff body $\left(\mathrm{L} / \mathrm{D}=6.48, \mathrm{Re}_{\mathrm{D}} \approx 2 \times 10^{5}\right)$, found that the most energetic structures in the wake are manifested as an unsteady vortex shedding with $m= \pm 1$ and $\mathrm{St}_{\mathrm{D}} \approx 0.2$, reminiscent of the symmetry-breaking laminar instabilities. The shedding is asymmetric and rotates randomly around the body's axis of revolution at a frequency of $S t_{D} \approx 0.002$. The reflectional symmetry, however, is still preserved if a rotating reference frame is considered. Two distinct time scales for the evolution of the flow can therefore be defined, similar to those proposed by Grandemange et al. ${ }^{18}$ when studying a rectilinear three dimensional body: a short time scale at $t$ $\approx 5 \mathrm{D} / \mathrm{V}_{\infty}$, associated with the vortex shedding, and a long time scale at $t \approx 5 \times 10^{2} \mathrm{D} / \mathrm{V}_{\infty}$, referring to the rotation of the reflectional symmetry plane of the shedding mode. In contrast to the wake generated by a rectilinear body, however, for an axisymmetric body, the wake does not switch between two symmetry-breaking states but moves between an infinite number of states depending on the azimuthal angle of the shedding.
The findings of Rigas et al. ${ }^{36}$ were confirmed by Gentile et al., ${ }^{15}$ using time-resolved particle image velocimetry (PIV) data. In this case, an axisymmetric body with $\mathrm{L} / \mathrm{D}=5$ was tested at $\mathrm{Re}_{\mathrm{D}}=6.7 \times 10^{4}$ performing stereoscopic PIV at different azimuthal planes downstream of the model base. The application of snapshot POD to the fluctuating part of the velocity field showed the existence of the same antisymmetric mode with $m= \pm 1$ described in Ref. 36 . This mode was found to be responsible for the long time instability of the reverse flow in the region close to separation, whereas further downstream it was linked to the fluctuations related to the development of the shear layer. A second mode pair with $m= \pm 2$, representing the "wake ovalisation," was also identified together with an axisymmetric mode with $m=0$ (visible only close to the model's base) describing the streamwise pulsation of the reverse flow region.

Although there seems to be agreement among researchers on the fact that the suppression of the very low frequency dynamics may be exploited to reduce drag, it is still not clear whether the suppression of this mode should aim at stabilising an axisymmetric state or a reflectional symmetry preserving state. For instance, Oxlade, ${ }^{31}$ controlling the dynamics of the wakes behind an axisymmetric body using synthetic jets actuated at different frequencies, reported that the configurations featuring the lowest drag were characterised by stable asymmetric wakes. An opposite trend was reported by Grandemange et al. ${ }^{20}$ and de la Cruz et al., ${ }^{9}$ where the lowest drag configurations were accompanied by the restoration of the axial symmetry.

It is evident that a lot of progress has been made in the understanding of the dynamic behavior of axisymmetric wakes, but there are unanswered questions. In particular, the effects produced by the very low frequency dynamics on the three-dimensional structure of the near-wake as a whole are still not clear (at least at higher Reynolds number). Neither are the factors leading to the selection of either an axisymmetric topology or a reflectional symmetry preserving state as low-drag conditions. The present manuscript seeks to provide an answer to these questions. The near-wake region of an axisymmetric body with $\mathrm{L} / \mathrm{D}=5$ has been investigated, using base pressure tappings and, for the first time, Tomographic Particle Image Velocimetry (TPIV). Pressure tappings over the entire model base are employed to validate the experimental setup against the existing literature ${ }^{36}$ and characterise the frequency content of the main unsteady flow features, isolated by applying Proper Orthogonal Decomposition (POD) to the base pressure dataset. The most coherent three dimensional unsteady structures are then isolated in the near-wake region by means of snapshot POD applied to the TPIV data. The relationship between the base drag and the wake dynamics is also investigated.

\section{EXPERIMENTAL METHODOLOGY}

\section{A. Experimental setup}

Testing was performed in the Loughborough University Large Wind Tunnel. This test facility features a closed test 
section and an open circuit with a horseshoe shaped layout. The tunnel has a test section of $1.92 \times 1.32 \times 3.6 \mathrm{~m}\left(\mathrm{~W}_{\mathrm{T}} \times \mathrm{H}_{\mathrm{T}}\right.$ $\left.\times L_{T}\right)$ and a contraction ratio of 7.3:1. Turbulence intensity is kept low by a honeycomb, used to straighten the flow, and a pair of turbulence reduction screens, located upstream of the contraction. Wind is generated through a $2.65 \mathrm{~m}$ diameter, variable-speed, fixed pitch fan, located downstream of the working section. More information can be found in the work of Johl et al. ${ }^{23}$ The operating velocity in the test section was $30 \mathrm{~m} / \mathrm{s}$ throughout, with a free stream turbulence intensity of $0.2 \%$ and a flow uniformity of $\pm 0.4 \%$ (in empty conditions). Optical access in the test section was provided through glass sidewalls and roof panels.

The model employed is a cylinder with an elliptical nose cone and a truncated flat base, as shown in Fig. 1(a). The diameter of the cylinder $\mathrm{D}$ is $160 \mathrm{~mm}$, and the overall length $\mathrm{L}$ is $800 \mathrm{~mm}$, resulting in a length-to-diameter ratio L/D of 5 . The Reynolds number based on model diameter is $R e_{D}$ $=3.2 \times 10^{5}$. A carborundum roughness strip, with a width of $10 \mathrm{~mm}$, was placed on the junction between the nose section and the main body to force boundary layer transition. The axisymmetric body was supported by a steel shaft, with a diameter of $20 \mathrm{~mm}$, enclosed in a NACA 0021 aerofoil, with a chord $c=135 \mathrm{~mm}$ (or 0.84D). The shaft was centred with the point of maximum thickness of the airfoil (at $0.3 \mathrm{c}$ ).

In order to minimise the effects of the supporting system on the wake dynamics, ${ }^{20}$ the wing was moved as far upstream of the base as possible, close to the forward joint, with the leading edge of the wing itself located $370 \mathrm{~mm}$ (or 2.31D) downstream of the tip of the model nose. The shaft was attached to a turntable equipped with an automated yaw mechanism capable of $\psi=360^{\circ}$ rotation with $\pm 0.1^{\circ}$ accuracy, while the pitch angle $\alpha$ was manually adjusted with an accuracy of $\pm 0.25^{\circ}$. This was performed considering a right-handed reference system located at the intersection between the supporting shaft and the turntable, $400 \mathrm{~mm}$ (or 2.5D) below the model axis $\left[\mathrm{X}^{*}, \mathrm{Y}^{*}, \mathrm{Z}^{*}\right.$ in Fig. 1(b)].

A coordinate system with origin in the centre of the model base is used for the presentation of the results; the $x^{*}$ axis is aligned with the flow in the streamwise direction, the $z^{*}$ axis is vertical, positive upwards, and the $y^{*}$ axis follows a right handed coordinate system [Fig. 1(b)]. All quantities presented throughout the paper have been normalised using the base diameter $D$ as the reference length and the value of the free stream velocity $\mathrm{V}_{\infty}$; they are denoted with the superscript * For the sake of clarity, the symbol "_" is used to indicate all the time averaged quantities, " $\sim$ " denotes the conditionally averaged quantities, whilst the subscript "LOM" is used to denote quantities extracted from low order models.

\section{B. Base pressure measurements}

The base of the model was populated with 49 pressure taps [see Fig. 2(a)]. One tap was located in the middle of the base, whilst the remaining 48 taps were distributed around three concentric circles, centred with the base and located, respectively, at $R^{*}=0.143, R^{*}=0.286$, and $R^{*}=0.429$, with $\mathrm{R}^{*}$ denoting the non-dimensional distance from the origin of the reference system centred with the model base $\left[x^{*}, y^{*}, z^{*}\right.$ in Fig. 1(b)]. The taps were spaced following the procedure described in Ref. 4; the surface of the model base was divided into 49 equal-area cells, and a tap was located at the centre of each cell. Since the low frequency dynamics of the wake generated by an axisymmetric body have been proven to be very sensitive to angular offsets of as little as $0.2^{\circ},{ }^{16}$ the model was adjusted around $\psi=0^{\circ}$ and $\alpha=0^{\circ}$ until an axisymmetric time averaged base pressure distribution was obtained. Pressure measurements were performed using a 64 channel miniature pressure scanner with a manufacturer quoted accuracy of $\pm 1.47 \mathrm{~Pa}$, placed inside the model. Data were recorded at a frequency $\left(f_{\mathrm{s}}\right)$ of $300 \mathrm{~Hz}$ in two successive $300 \mathrm{~s}$ acquisitions to give a total sampling time of $t_{\mathrm{s}}=600 \mathrm{~s}$, corresponding to 1 , $125 \times 10^{5}$ convective units $t^{*}$, with $t^{*}=t \cdot V_{\infty} / D$. The freestream dynamic and static pressures were acquired at the start of the test section, $2430 \mathrm{~mm}$ upstream of the centre of the turntable. The pressure coefficients have been calculated using Eq. (1),

$$
\mathrm{C}_{\mathrm{P}}(\mathrm{t})=\frac{p(\mathrm{t})-p_{\infty}}{0.5 \rho \mathrm{SV}_{\infty}^{2}}
$$

where $p_{\infty}$ is the free stream static pressure, $\rho$ refers to the air density, and $\mathrm{S}$ is the base area $\left(\mathrm{S}=0.020 \mathrm{~m}^{2}\right)$. The base drag has then been estimated by integrating the pressure field,

$$
\mathrm{C}_{D_{\text {Base }}}(\mathrm{t})=-\frac{1}{\mathrm{~S}} \iint_{\mathrm{S}} \mathrm{C}_{p}(\mathrm{t}) d \mathrm{~S} \approx-\frac{1}{\mathrm{~S}} \sum_{i=1}^{\mathrm{N}_{\text {tap }}} \mathrm{C}_{p_{i}}(\mathrm{t}) \mathrm{S}_{i},
$$

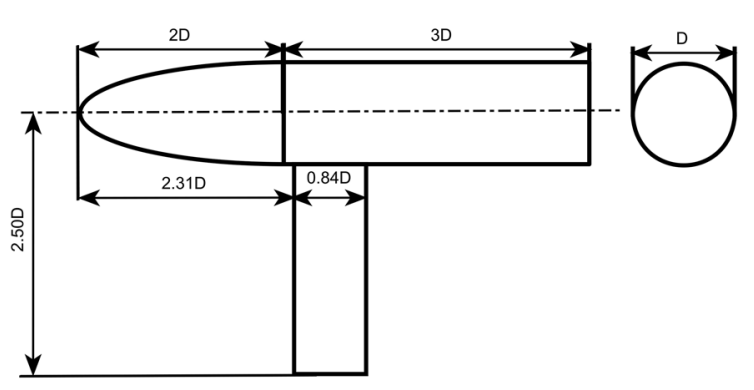

(a)

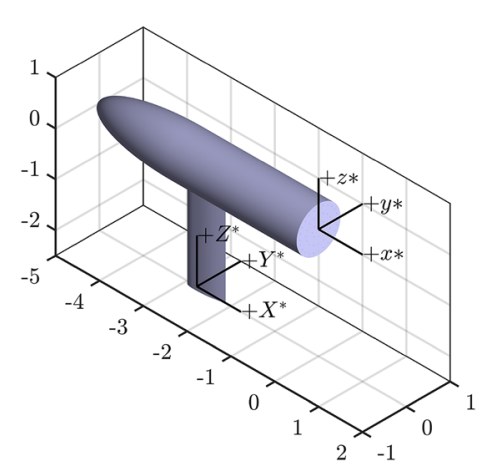

(b)
FIG. 1. (a) Representation of the axisymmetric body used in the present work. (b) Model mounting structure and reference systems. $X^{*}, Y^{*}, Z^{*}$ refer to the body's global reference system, whereas $x^{*}, y^{*}$, $z$ denote the reference system centred with the model base, used for the presentation of the results. The coordinates have been normalised using the base diameter $D$ as the reference length. 


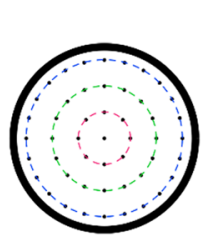

(a)

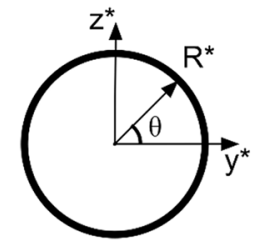

(b) (c)
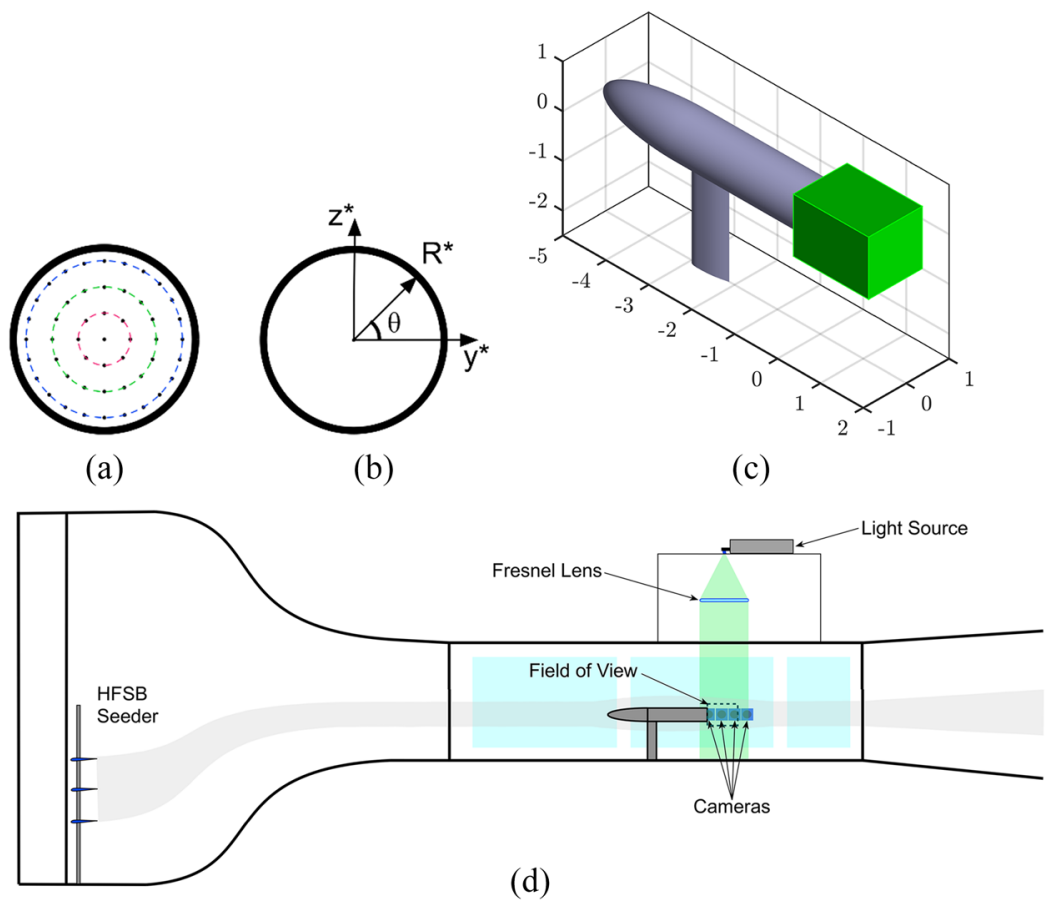

FIG. 2. (a) Representation of the distribution of the pressure taps on the model base. The dashed circles denote three concentric rings of taps located, respectively, at $R^{*}=0.143$ (red circle), $R^{*}=0.286$ (green circle), and $R^{*}=0.429$ (blue circle), from the centre of the base. (b) Polar reference system used to present some of the results. (c) Field of view considered in the TPIV acquisitions. (d) Schematic representation of the TPIV setup. where $C_{p_{i}}(t)$ is the value of the pressure coefficient recorded by the $i$ th tap and $S_{i}$ is the area associated with the same tap (with $\mathrm{S}_{i}=\mathrm{S} / \mathrm{N}_{\text {taps }}$ ).

Following the approach proposed by Rigas et al., ${ }^{36}$ further insights into the wake symmetry and its temporal evolution have been gathered by considering the location of the centre of pressure, defined as

$$
\operatorname{CoP}(t)=\frac{\iint_{S} C_{p}(t) \mathbf{x} d S}{\iint_{S} C_{p}(t) d S},
$$

with $\mathbf{x}=\left(y^{*}, z^{*}\right)$ in the Cartesian coordinate system of the base. The results have then been projected on a polar coordinate system [Fig. 2(b)].

Proper Orthogonal Decomposition (POD) has been used, with the aim of isolating the main features of the unsteady pressure field. Following the method proposed by Lumley, ${ }^{26}$ a generic dataset $\mathbf{F}(\mathbf{x}, \mathbf{t})$ is decomposed as

$$
\mathbf{F}(\mathbf{x}, \mathrm{t})=\mathbf{F}_{0}(\mathbf{x})+\mathbf{f}^{\prime}(\mathbf{x}, \mathrm{t})=\mathbf{F}_{0}(\mathbf{x})+\sum_{n=1}^{N_{t}} \boldsymbol{\Phi}_{n}(\mathbf{x}) a_{n}(\mathrm{t}),
$$

where $\mathrm{N}_{t}$ indicates the number of measurement points in time, $\mathbf{F}_{0}$ indicates the mean of the considered field, and $\mathbf{f}^{\prime}(\mathbf{x}, t)$ indicates its fluctuating components. $a_{n}(t)$ refers to the temporal coefficients defined in Eq. (9). The basis functions $\boldsymbol{\Phi}_{n}(\mathbf{x})$ are the so-called "POD modes." They are defined as the eigenfunctions of the covariance matrix $\mathbf{R}$,

$$
\mathbf{R}=\mathbf{X} \cdot \mathbf{X}^{\mathrm{T}}
$$

where $\mathbf{X}$ is a matrix with

$$
\mathbf{X}=\left[\mathbf{f}\left(\mathbf{x}, t_{1}\right), \mathbf{f}\left(\mathbf{x}, t_{2}\right), \ldots, \mathbf{f}\left(\mathbf{x}, t_{n}\right)\right] \in \mathbf{R}^{N_{s} \times N_{t}}
$$

and $N_{s}$ refers to the number of points sampled in space. The basis functions $\boldsymbol{\Phi}_{n}(\mathbf{x})$ can therefore be determined by solving the eigenvalue problem,

$$
\mathbf{R} \boldsymbol{\Phi}_{n}=\lambda_{n} \boldsymbol{\Phi}_{n}, \quad \boldsymbol{\Phi}_{n} \in \mathbf{R}^{\mathrm{N}_{s}},
$$

with $\lambda_{1} \geq \cdots \geq \lambda_{n}$. The eigenvalues $\lambda_{n}$ associated with the POD modes are representative of the energy content $E$ of the fluctuations captured by each mode. A low order model can then be written as

$$
\mathbf{F}(\mathbf{x}, t) \approx \mathbf{F}_{0}(\mathbf{x})+\sum_{n=1}^{M} \boldsymbol{\Phi}_{n}(\mathbf{x}) a_{n}(t),
$$

with $\mathrm{M}<\mathrm{N}_{t}$ and the temporal coefficients $a_{n}(t)$ determined projecting each spatial mode $\boldsymbol{\Phi}_{n}$ on the original dataset $\mathbf{f}(\mathbf{x}, t)$,

$$
a_{n}(t)=\left\langle\left(\boldsymbol{\Phi}_{n}(\mathbf{x})\right)^{\mathrm{T}}, \mathbf{f}(\mathbf{x}, t)\right\rangle .
$$

Since the frequency resolution of the pressure measurements was high enough to resolve most of the characteristic temporal scales of the flow field, the Power Spectral Density (PSD) of the POD temporal coefficients has been determined. The Welch periodogram described in Ref. 43 has been used in this case, considering intervals of $10.0 \mathrm{~s}$, with a $50 \%$ overlap. The PSD has been calculated separately for each interval applying a Hann window. ${ }^{21}$ The same methodology has also been used to calculate the spectrum of the pressure signal as recorded by using the taps. 
In some cases, the coherence between signals recorded by using taps placed in different locations of the model base has also been considered, following the approach proposed by Duell and George. ${ }^{10}$ The magnitude of the coherence between two generic synchronised signals $i$ and $j$ has been calculated according to the equation

$$
\Lambda_{\mathbf{i j}}(f)=\frac{\left|\mathbf{P}_{\mathbf{i j}}(f)\right|^{2}}{\mathbf{P}_{\mathbf{i i}}(f) \cdot \mathbf{P}_{\mathbf{j j}}(f)},
$$

where $\mathbf{P}_{\mathbf{i j}}(f)$ is the cross-spectrum and $\mathbf{P}_{\mathbf{i i}}(f)$ and $\mathbf{P}_{\mathbf{j} \mathbf{j}}(f)$ are the auto-spectra of the signals. The phase relationship between the two signals has then been determined as

$$
\chi_{\mathbf{i j}}(f)=\left|\tan ^{-1}\left(\frac{\operatorname{Im}\left(\mathbf{P}_{\mathbf{i j}}(f)\right)}{\operatorname{Re}\left(\mathbf{P}_{\mathbf{i j}}(f)\right)}\right)\right|,
$$

where $\operatorname{Im}\left(\mathbf{P}_{\mathbf{i j}}(f)\right)$ and $\operatorname{Re}\left(\mathbf{P}_{\mathbf{i j}}(f)\right)$ denote the imaginary part and the real part of the cross-spectrum, respectively. For the sake of simplicity, the absolute value of the phase angle is considered.

\section{Tomographic PIV}

Tomographic particle image velocimetry ${ }^{37}$ was used in order to characterise the three dimensional topology of the recirculating region behind the axisymmetric body. A volumetric field of view measuring $280 \times 200 \times 200 \mathrm{~mm}\left(\mathrm{~L}_{\text {TPIV }}\right.$ $\times \mathrm{W}_{\text {TPIV }} \times \mathrm{H}_{\text {TPIV }}$ ) was used to capture the near-wake region, whose size had been determined previously using planar PIV data [Fig. 2(c)].

Four LaVision ${ }^{\circledR}$ dual frame cameras were used during the acquisition, consisting of two LaVision ${ }^{\circledR}$ Imager Pro X $4 \mathrm{M}$ cameras (with a resolution of $2048 \times 2048$ pixels and a pixel size of $7.4 \mu \mathrm{m}^{2}$ ) and two LaVision ${ }^{\circledR}$ sCMOS cameras (with a resolution of $2560 \times 2160$ pixels and a pixel size of $\left.6.5 \mu \mathrm{m}^{2}\right)$. The cameras were mounted in a horizontal arrangement, on a frame placed outside the tunnel test section. The aperture angle of the system was greater than $60^{\circ} .{ }^{37}$ Each camera was equipped with a Nikon ${ }^{\circledR}$ lens, with a focal length of $50 \mathrm{~mm}$, mounted on a tilt system in order to satisfy the Scheimpflug criterion. ${ }^{35}$ An aperture of $f^{\#}=8$ was used on all of the cameras to maintain the focus throughout the volume considered. This resulted in a depth of field between $\approx 210 \mathrm{~mm}$ and $\approx 310 \mathrm{~mm}$, depending on the camera. Calibration was carried out using a 3D calibration plate. The 3D mapping functions obtained were then corrected by applying the volume selfcalibration procedure, ${ }^{45}$ resulting in a final RMS error of less than 0.03 pixels.

Helium filled soap bubbles, with a diameter of $300 \mu \mathrm{m}$, were used to seed the flow. ${ }^{8}$ The bubbles were released from 30 nozzles producing 40000 bubbles per second each. The nozzles were hosted inside 3 wing shaped seeding rakes, horizontally mounted on a purpose built frame located at the start of the contraction, in order to minimise the aerodynamic interference with the model mounted downstream in the test section [Fig. 2(d)]. This allowed a stream-tube with a square cross section of $\approx 220 \times 220 \mathrm{~mm}$ (in the test section) to be seeded. With the seeding system in place, a limited increase in the free stream turbulence intensity was measured in proximity to the model axis (at $\mathrm{X}^{*}=0, \mathrm{Y}^{*}=0, \mathrm{Z}^{*}=2.5 \mathrm{D}$ and no model in place), from $\approx 0.2 \%$ to $\approx 0.4 \%$. Approximately 200000 bubbles were captured in each $2 \mathrm{D}$ image, giving a particle density of about $0.04 p p p$ (the particle size was $\approx 6$ pixels, with an intensity profile featuring a Gaussian shape). Combined with the number of cameras (4) used in the present experiment, this results in a quality factor $Q \approx 0.95 .{ }^{37}$

The particles were illuminated using a $200 \mathrm{~mJ}$ Nd:YAG laser. The laser was expanded through a volume optic with a focal length of $f^{\#}=-12$. The resulting cone of light was then collimated using a Fresnel lens to suppress the tails of the Gaussian beam profile, keeping the edges of the measurement volume well defined. The approximate diameter of the resulting column of light was $\approx 400 \mathrm{~mm}$ [Fig. 2(d)]. Two consecutive acquisitions were performed, each consisting of 250 statistically independent image pairs, captured at a sampling frequency of $f_{\text {TPIV }}=7.26 \mathrm{~Hz}$, with an inter-frame time of $80 \mu \mathrm{s}$. This resulted in a total sampling time of $68.9 \mathrm{~s}$ corresponding to $1.291 \times 10^{4}$ convective units $t^{*}$. Since the image noise has been proven to be a critical factor in determining the quality of the $3 \mathrm{D}$ particle reconstruction, ${ }^{17}$ the raw images were pre-processed by performing a background intensity subtraction, based on the average intensity of each dataset. The particles were then further isolated from the background by subtracting a constant intensity of 65 counts to eliminate dimmer objects. 27 The "FastMART" algorithm implemented in the LaVision ${ }^{\circledR}$ DaVis 8.4 software was used to reconstruct the position of the particles in the three dimensional space. This algorithm is a computationally more efficient implementation of the simultaneous MART algorithm described in Ref. 2. The quality of the reconstruction was assessed by evaluating the signal-to-noise ratio (Fig. 3), which in the present experiment was found to be greater than $2 .{ }^{27}$ The processing to produce the vector field was performed using a direct correlation multi-pass technique, starting with an interrogation window measuring $160 \times 160 \times 160$ voxels and decreasing in size to 128,104 , and then finally 96 voxels (with approximately

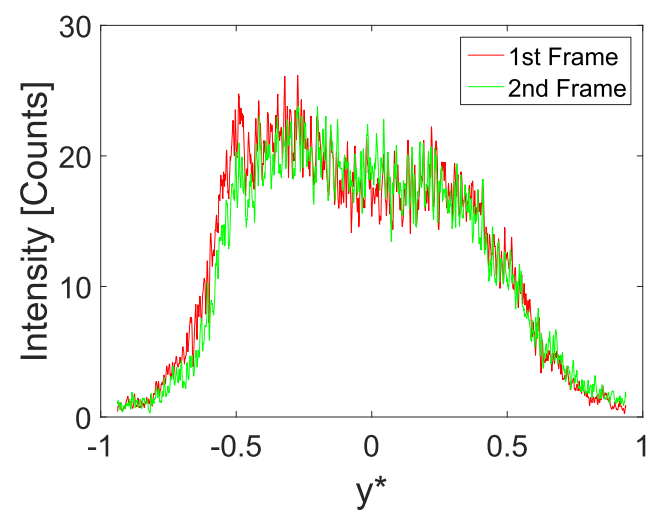

FIG. 3. Light intensity profile measured across the reconstructed volume. The values are the result of the average of 10 snapshots. 


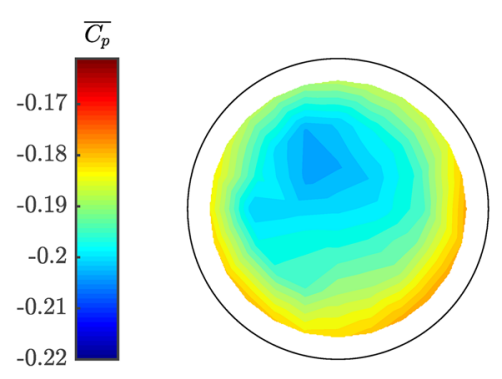

(a)

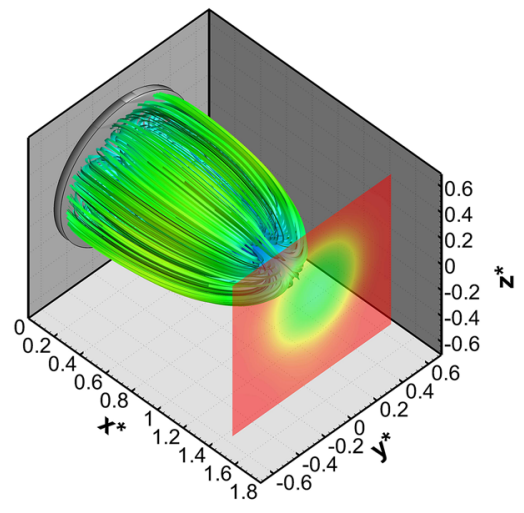

(b)

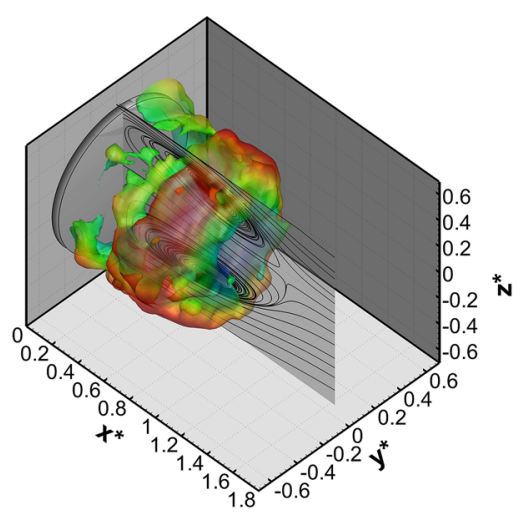

(d)

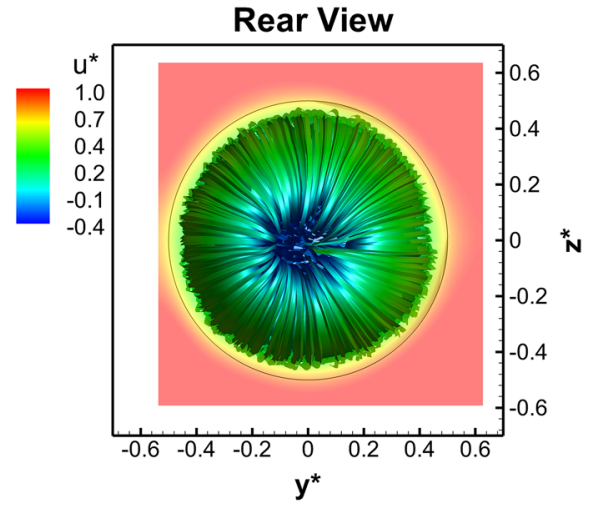

(c)

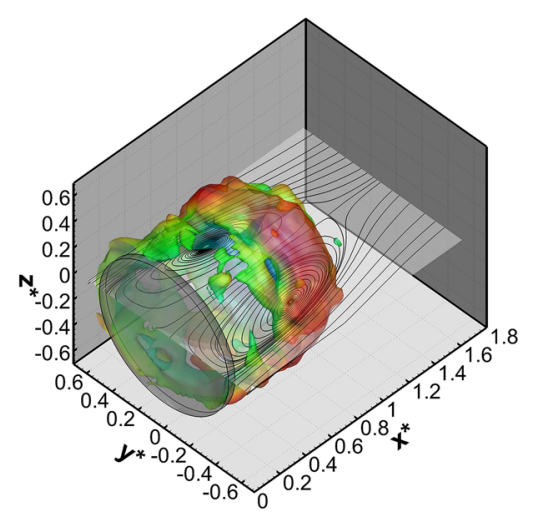

(e)

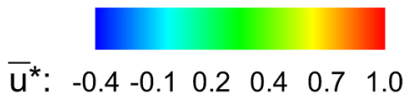

FIG. 4. Time averaged results: (a) base pressure distribution; (b) near-wake topology; (c) near-wake topology as seen from a point of view located downstream of the wake closure. The recirculating flow is visualised by means of stream ribbons coloured with the non-dimensional values of the axial component of the velocity $\overline{u^{*}}$. For the sake of clarity, only the ribbons with $\overline{u^{*}}<0.5$ have been considered. The continuous line in (a) denotes the location of the model trailing edge. [(d) and (e)] Iso-surfaces of $\lambda_{2}^{*}=-0.25$ coloured with $u^{*}$; the $2 \mathrm{D}$ streamlines refer to the mid-vertical plane $(\mathrm{d})$ and the mid-horizontal plane $(\mathrm{e})$

4 particles captured in each interrogation volume). A single pass was performed on the first two steps, and volume binning was applied to reduce processing time. Two passes were used for the final two steps with no volume binning, while overlap was kept constant at $75 \%$. The velocity data obtained were then post-processed using a universal outlier detection technique to remove spurious vectors ${ }^{44}$ as well as a smoothing function with a $3 \times 3 \times 3$ spatial kernel. The resulting vector field is characterised by a resolution of $3.5 \mathrm{~mm}^{3}$ and a total vector count of 255000 . The level of uncertainty associated with each dataset has been estimated considering a 95\% confidence level, as in the work of Perry et $a .^{34}$ A value of $\approx 0.3 \%$ has been obtained for the free-stream velocity. The level of uncertainty, however, is seen to increase up to more than $5 \%$ in the shear layers, in agreement with that found by Sellappan et al. ${ }^{38}$ A similar error is also observed at the corners of the volumetric field of view, as a consequence of the worsening of the signal-to-noise ratio (Fig. 3). As such, the latter regions have not been considered for the unsteady analysis, in order to avoid any bias in the results.

As for the base pressure, the main unsteady features of the velocity field have been captured by applying POD to the resulting vector fields. The snapshot method developed by Sirovich ${ }^{40}$ has been applied in this case due to its higher computational efficiency when the temporal domain is much smaller than the spatial domain $\left(N_{t} \ll N_{s}\right)$. This method relies on solving an eigenvalue problem of smaller size,

$$
\mathbf{X}^{\mathrm{T}} \mathbf{X} \mathbf{A}_{n}=\lambda_{n} \mathbf{A}_{n}, \quad \mathbf{A}_{n} \in \mathbf{R}^{\mathrm{N}_{t}}
$$

with $\mathbf{X}$ defined as in Eq. (6). The POD modes $\boldsymbol{\Phi}_{n}$ are then given by 


$$
\boldsymbol{\Phi}_{n}=\frac{1}{\sqrt{\lambda_{n}}} \mathbf{X A}_{n} \in \mathbf{R}^{\mathrm{N}_{\mathrm{s}}}, \quad n=1,2, \ldots, \mathrm{N}_{t},
$$

whilst the temporal coefficients $a_{n}(\mathrm{t})$ can still be determined using Eq. (9). Examples of applications of POD to the investigation of turbulent flows can be found in the work of Alfonsi ${ }^{1}$ and Semeraro et al. ${ }^{39}$

\section{RESULTS AND DISCUSSION}

\section{A. Time averaged results}

The time averaged flow field, presented in Fig. 4, shows that the model has an almost axisymmetric wake, as it was tested at $\psi \approx 0^{\circ}, \alpha \approx 0^{\circ}$. The contour plot of base pressure, averaged over $600 \mathrm{~s}$, shows a region of constant low pressure centred with the base and pressure recovery occurring close to the outer edge of the body [Fig. 4(a)]. The pressure field is uniform in the azimuthal direction with a positive pressure gradient in the radial direction (from the centre toward the outer edge of the base). This is in good agreement with that reported by Rigas et al. ${ }^{36}$ for a similarly shaped body. The slightly higher eccentricity seen in the time averaged pressure distribution in this case may be a consequence of the much shorter sampling time considered in the present investigation $\left(t_{\mathrm{s}}=600 \mathrm{~s}\right.$ compared to $t_{\mathrm{s}}=1.92 \times 10^{4} \mathrm{~s}$ in Ref. 36$)$ as well as the presence of some small residual asymmetries in the experimental setup. A time averaged base drag value of $\bar{C}_{D_{\text {Base }}}=0.189$ has been calculated in these conditions.

Similar descriptions can be applied to the time averaged topology of the recirculating region [Figs. 4(b) and 4(c)]. A good level of symmetry can be seen in the near-wake region, in good agreement with the results of the findings of Gentile et al. ${ }^{15}$ Indeed, the application of the $\lambda_{2}$ criterion $^{22}$ to the same field reveals the existence of a well defined torus located between the base and the wake closure, as can be seen in Figs. 4(d) and $4(\mathrm{e})$.

\section{B. Unsteady results}

The similarities with the results reported in the studies of Rigas et $a l .{ }^{36}$ and Gentile et al. ${ }^{15}$ extend, with some minor differences, to the unsteady flow field (Fig. 5). The root mean square of the pressure fluctuation $\left(\operatorname{RMS}\left(C_{p}^{\prime}\right)\right)$ recorded on the base [Fig. 5(a)] is seen to reach its highest values close to the centre and then decreases noticeably when moving toward the trailing edge of the model. A similar trend was reported by Rigas et al., ${ }^{36}$ although in that case the maximum values of $\operatorname{RMS}\left(C_{p}^{\prime}\right)$ were located at $\mathrm{R}^{*} \approx 0.15$, resulting in a characteristic annular distribution. Such a distribution is not seen in the present case, arguably because of the coarser grid of taps used. It is however observed in the contour plots obtained for the axial component of the Reynolds stresses $\left(\mathrm{T}_{u u}^{*}\right)$ calculated at $x^{*}=0.75$ and $x^{*}=1.50$ [Figs. 5(b) and 5(e)]. This is consistent with the presence of strong coherent motions of the flow reversal, as pointed out by Grandemange et al. ${ }^{18}$ and Pavia and Passmore 32 in the case of rectilinear threedimensional bodies. A different distribution characterises the other two normal components of the Reynolds stress tensor $\left(\mathrm{T}_{v v}^{*}\right.$ and $\left.\mathrm{T}_{w w}^{*}\right)$, which tend to concentrate mainly around the body's axis of symmetry [Figs. 5(c), 5(d), 5(f), and 5(g)].

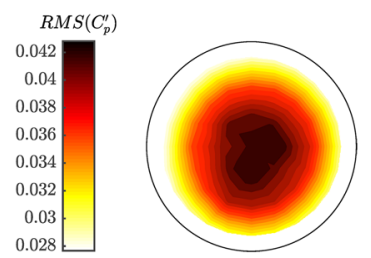

(a)

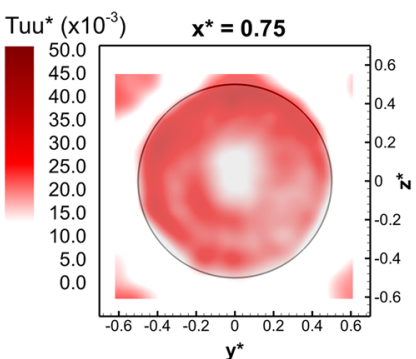

(b)

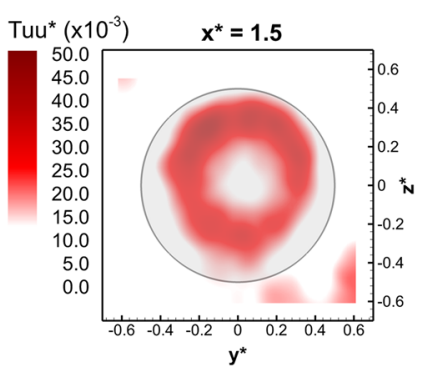

(e)

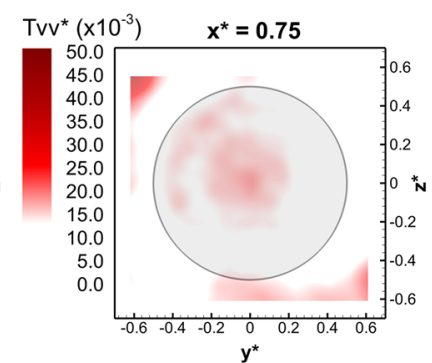

(c)

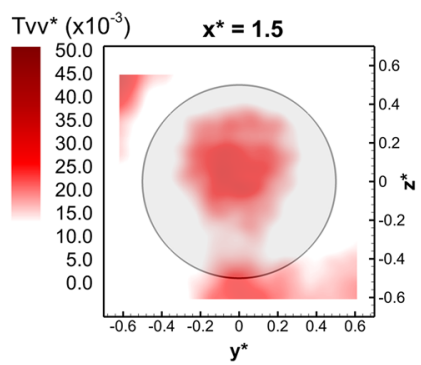

(f)

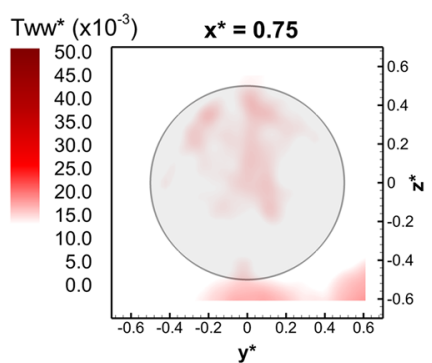

(d)

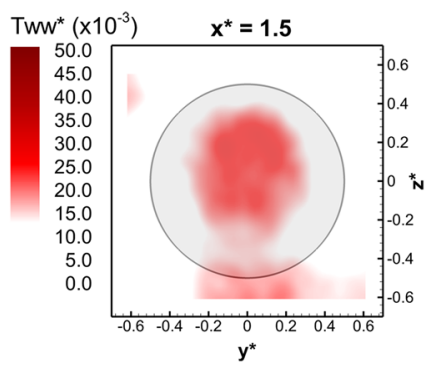

(g)

FIG. 5. (a) Root mean square of the pressure fluctuation recorded over the model base. [(b)-(g)] Contour plots of the normal components of the normalised Reynolds stresses extracted from two different cross-planes located downstream of the model base, respectively, at $x^{*}=0.75[(\mathrm{~b})-(\mathrm{d})]$ and $x^{*}=1.50[(\mathrm{e})-(\mathrm{g})]$. 
Furthermore, they appear to gain strength when approaching the wake closure (at $x^{*}=1.50$ ), where flapping motions induced by the shear layer instabilities are stronger. ${ }^{15}$ Non-negligible values of $\mathrm{T}_{v v}^{*}$ and $\mathrm{T}_{w w}^{*}$ are also seen in the lower portion of the field of view, as a result of the interactions between the main body and the supporting wing. These values are still "physical," unlike those seen for the same quantities (including $\mathrm{T}_{u u}^{*}$ ) at the corners of all cross-planes reported in Fig. 5, which are a consequence of the increased level of noise that characterises the outermost regions of the TPIV domain (Sec. II C). These areas have therefore been excluded from any further unsteady analysis.

\section{Characterisation of the wake dynamics}

The application of proper orthogonal decomposition (see Sec. II B) to the base pressure field yields the spatial modes $\phi_{n}$ reported in Fig. 6, along with the related energy content. The first five modes are sufficient to capture about $69 \%$ of the energy associated with the pressure fluctuation recorded over the entire model base, with the first three modes alone accounting for almost $58 \%$. This value is $\approx 15 \%$ points lower than that reported in the work of Rigas et al., ${ }^{36}$ arguably due to the higher Reynolds number $\left(\operatorname{Re}_{\mathrm{D}}=3.2 \times 10^{5} \mathrm{com}-\right.$ pared to $R e_{\mathrm{D}}=2 \times 10^{5}$ ) and the shorter acquisition time $\left(t_{\mathrm{s}}=600 \mathrm{~s}\right.$ compared to $\left.\mathrm{s}=1.92 \times 10^{4} \mathrm{~s}\right)$ considered in the present work. Remarkable similarities between the two experiments, however, can be seen in the shapes of the first five spatial modes as well as the spectral content of the related temporal coefficients $a_{n}$ (Fig. 6). In both cases, the highest amount of energy is captured by a pair of modes whose spatial functions are characterised by an azimuthal wave number $m= \pm 1$ [Figs. 6(a) and 6(b)], while the premultiplied spectra of the related temporal coefficients $\left[a_{1}\right.$ and $a_{2}$ in Fig. $\left.6(\mathrm{f})\right]$ show a broad peak at very low frequency $\left(\mathrm{St}_{\mathrm{D}} \approx 0.002\right)$ reminiscent of the long-time erratic motion of the flow reversal described also in the work of Gentile et al. ${ }^{15}$ Two additional peaks are visible in the spectra associated with these temporal coefficients. These two peaks are narrower than that seen at $\mathrm{St}_{\mathrm{D}} \approx 0.002$; their energy is therefore lower. The peak associated with the higher frequency content is located at $\mathrm{St}_{\mathrm{D}}$ $=0.235$. A similar peak is often reported in the literature for axisymmetric bodies ${ }^{19,20}$ as well as 3D rectilinear bluff bodies $^{18,42}$ and is associated with flapping motions of the wake. A second peak, present at $S t_{D}=0.095$, becomes the predominant unsteady feature when the spectra related to the fourth and fifth POD modes are considered [ $a_{4}$ and $a_{5}$ in Fig. 6(f)]. As in the case of the first two modes, these two modes form a pair, since they share the same spectral content, and have similar energy levels, and their spatial functions, characterised by an azimuthal wave number $m= \pm 2$ [Figs. 6(d) and 6(e)], are in quadrature. The ratio of the frequencies at $\mathrm{St}_{\mathrm{D}}=0.235$ and $\mathrm{St}_{\mathrm{D}}=0.095$ suggests that they are not harmonics, as postulated by Rigas et al. ${ }^{36} \mathrm{~A}$ further argument to support this thesis is that the wave number associated with the spatial functions obtained for the mode at lower frequency $\left(\mathrm{St}_{\mathrm{D}}=0.095\right)$ is higher than that reported for the motion at higher frequency $\left(\mathrm{St}_{\mathrm{D}}=0.235\right)$, with $m= \pm 2$ in the former case and $m= \pm 1$ in the latter. A peak at $\mathrm{St}_{\mathrm{D}}=0.08$ is seen instead for the third mode $\left[a_{3}\right.$ in Fig. 6(f)], whose spatial function features a characteristic $m=0$ distribution [Fig. 6(c)]. For such reasons, this peak can be associated with the axisymmetric pulsation usually known as "bubble pumping." In the same spectrum, the peak at $\mathrm{St}_{\mathrm{D}} \approx 0.23$ can also still be seen, although with a much lower energy content than that observed in the first pair of modes.

Additional information on the wake dynamics can be extracted by applying POD to the TPIV data, following the methodology described in Sec. II C. The first pair of modes extracted from the three-dimensional velocity field [Figs. 7(a) and 7(b)] is characterised by the same $m \pm 1$ azimuthal number seen in the base pressure data, but at $11.5 \%$ the combined turbulent kinetic energy is noticeably lower than the fluctuating energy captured by the similar modes in the base pressure

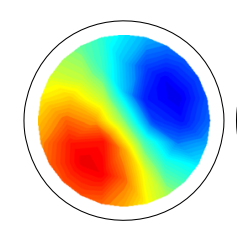

(a)

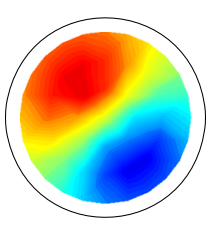

(b)

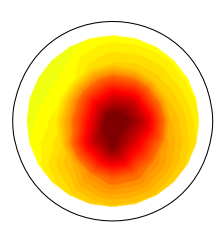

(c)

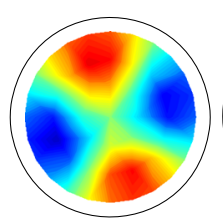

(d)

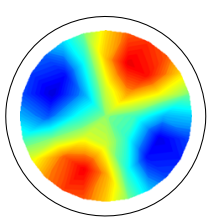

(e)

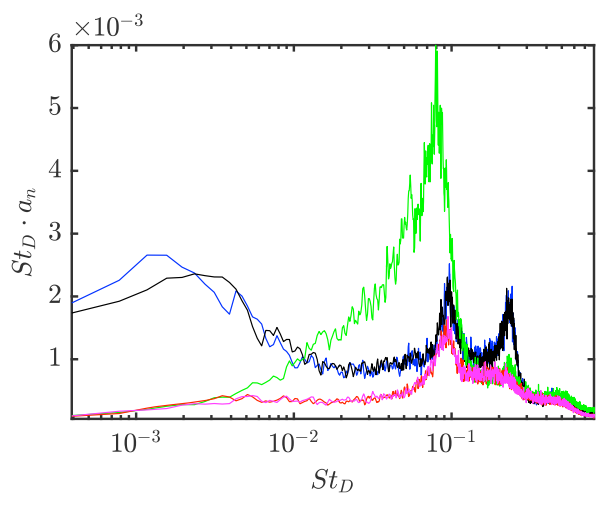

(f)
FIG. 6. [(a)-(e)] Spatial functions associated with the first five POD modes extracted from the base pressure data (the continuous line indicates the location of the model trailing edge). (f) Premultiplied spectra of the temporal coefficients associated with the same modes. The energy content associated with each mode is: (a) $\phi_{1_{\mathrm{Cp}}}-22.4 \%$. (b) $\phi_{2 \mathrm{Cp}}-21.6 \%$. (c) $\phi_{3 \mathrm{Cp}}-13.8 \%$. (d) $\phi_{4_{\mathrm{Cp}}}-5.5 \%$. (e) $\phi_{5_{\mathrm{Cp}}}-5.5 \%$. 

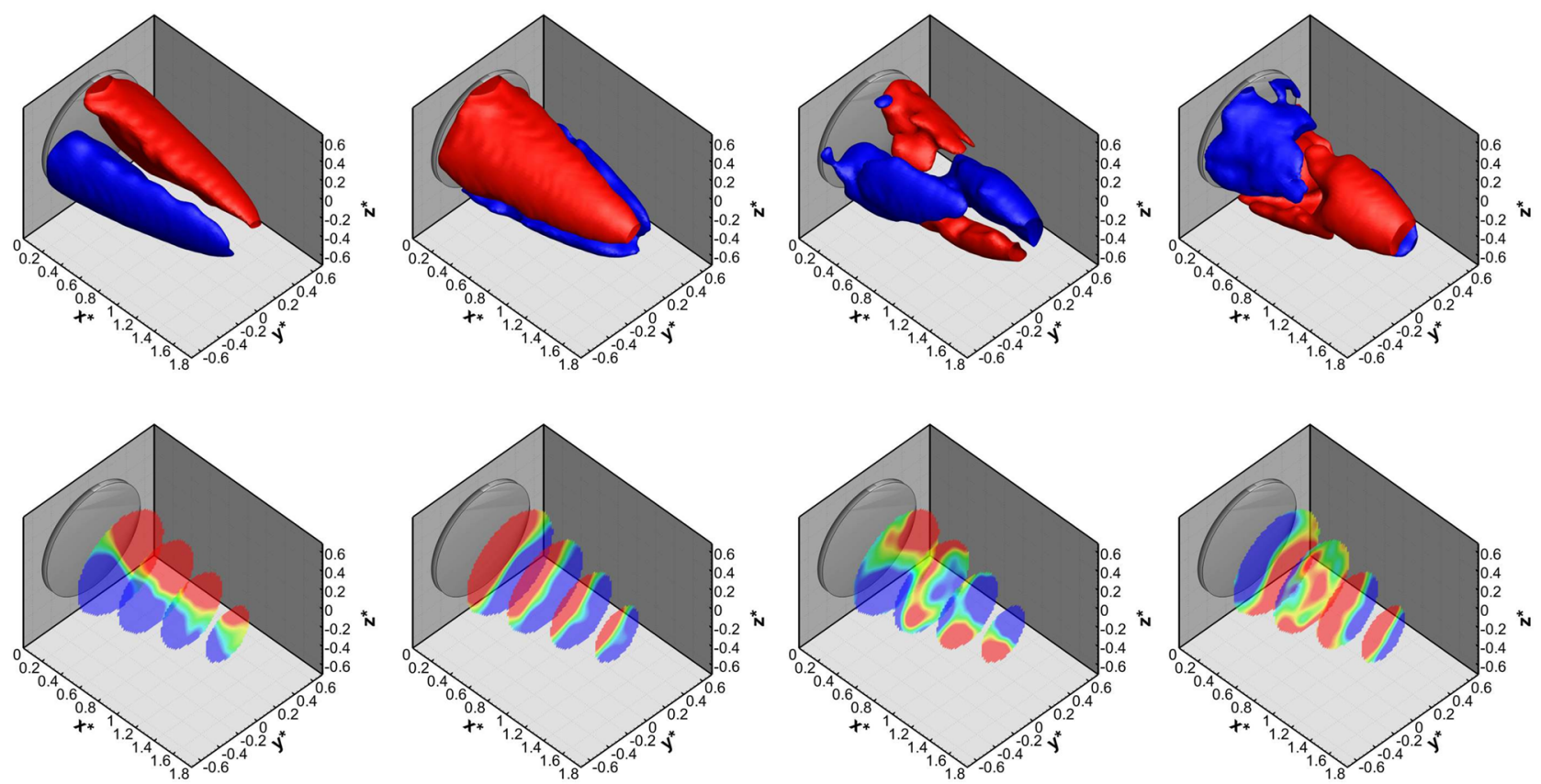

(a)

(b)

(c)

(d)

$$
\phi_{\mathrm{nu}}\left(\mathrm{x} 10^{-3}\right):-\begin{array}{rlllll}
-1.0 & -0.6 & -0.2 & 0.2 & 0.6 & 1.0
\end{array}
$$

FIG. 7. Spatial functions of the first [(a) and (b)] and second pairs [(c) and (d)] of POD modes extracted from the TPIV dataset. Contour plot and iso-surfaces performed considering the axial component of $\phi_{n_{V^{*}}}$. The iso-surfaces have been smoothed for better visualisation; for the same reason, only the portion of the domain with $\overline{u^{*}}<0.5$ has been considered. The 2D slices are located at $x^{*}=0.375, x^{*}=0.75, x^{*}=1.125$, and $x^{*}=1.5$. The energy level associated with each mode is: (a) $\phi_{1 V}-6.72 \%$. (b) $\phi_{2 \mathrm{~V}}-4.82 \%$. (c) $\phi_{3_{\mathrm{V}}}-1.75 \%$. (d) $\phi_{4_{\mathrm{V}}}-1.74 \%$.

dataset. This difference is arguably due to the effects of the shear layer unsteadiness (not captured by the pressure tappings) that tends to spread the values of the fluctuating energy over a higher number of modes. Each mode is characterised by the presence of two opposite regions with an excess and a deficit of streamwise momentum. The zones of opposite streamwise velocity fluctuation extend for the entire length of the wake and are associated with the motion of the rear recirculation. The amplitude of this motion is larger close to the base than in proximity to the wake closure, where the effects of the shear layer unsteadiness become predominant. ${ }^{15}$

The effect of these modes on the wake dynamics becomes clearer when a low order model is considered. The model was built combining the first two POD modes with the time averaged flow field described in Sec. III A, according to the equation

$$
\mathrm{V}_{\mathrm{LOM}_{2}}\left(x^{*}, t^{*}\right)=\overline{\mathrm{V}}\left(x^{*}\right)+\sum_{n=1}^{\mathrm{M}} a_{n_{\mathrm{V}}}\left(t^{*}\right) \phi_{n_{\mathrm{V}}}\left(x^{*}\right)
$$

The results, presented in Fig. 8 for four different snapshots referring to non-consecutive instants $t^{*}$ (with $\Delta t_{\text {TPIV }}^{*}=V_{\infty} /(D$. $\left.f_{\text {TPIV }}\right)$ ), are consistent with those reported in the work of
Rigas et $a l .{ }^{36}$ and Gentile et al. ${ }^{15}$ They highlight the existence of a Reflectional Symmetry Preserving (RSP) state consisting of a hairpin vortex that is shed from the trailing edge at different random azimuthal positions. The vortex extends for $\theta_{v} \approx \pi \mathrm{rad}$ in the azimuthal direction close to the base and has two "tails" that bend toward the "opposite side" (at $\approx \theta_{v}$ $+\pi \mathrm{rad}$ ) while moving downstream, forming a pair of (weak) counter-rotating streamwise vortices. This structure is similar to that proposed by Taneda ${ }^{41}$ for the wake of a sphere and most recently by Pavia et al. ${ }^{33}$ for the wake generated by a simplified automotive shape tested at a similar Reynolds number.

This structure becomes easier to visualise when the $\lambda_{2}$ criterion $^{22}$ is applied to the same field. The results, illustrated with iso-surfaces of $\lambda_{2}{ }^{*}=-0.25$ coloured with the values of $u_{\mathrm{LOM}}^{*}$, are presented in Fig. 9 and its Multimedia view, for four consecutive snapshots. The hairpin vortex connects the transverse structure forming close to the model base (the "head") with the longitudinal structures shed downstream of the wake closure (the "tails"), as highlighted by the dashed line drawn in Figs. 9(a), 9(c), and 9(d). These structures, indeed, appear to be dynamically linked together, as they are 


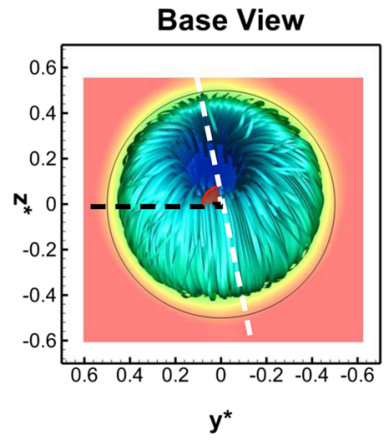

Rear View

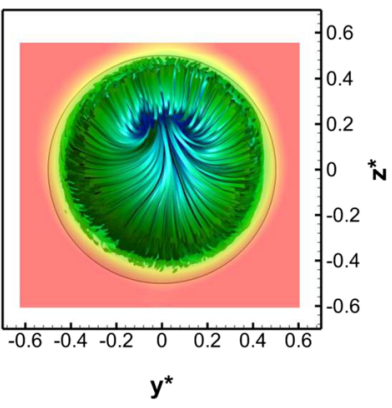

(a)

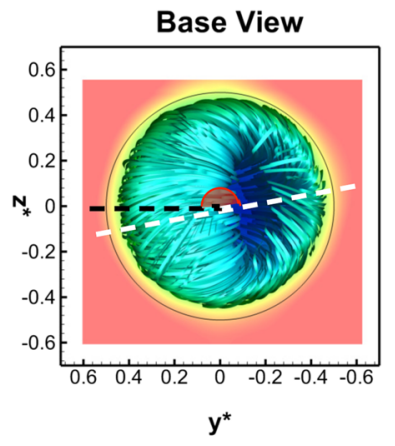

Rear View

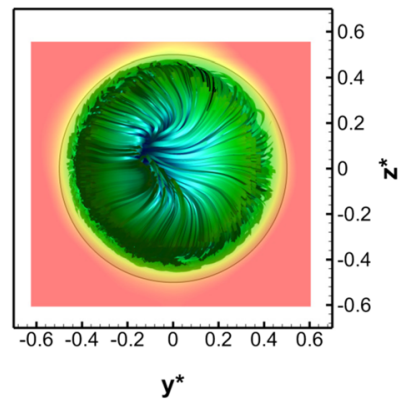

(b)

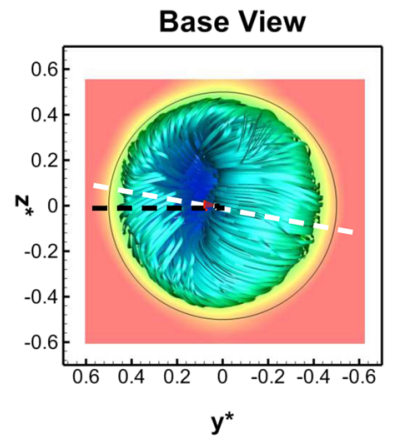

Rear View

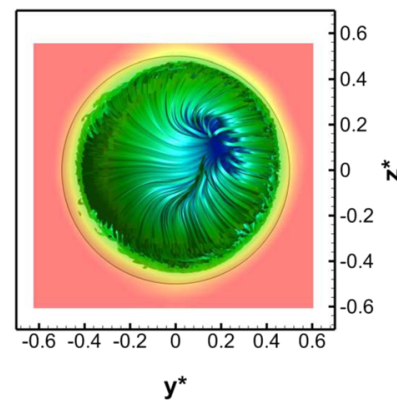

(c)
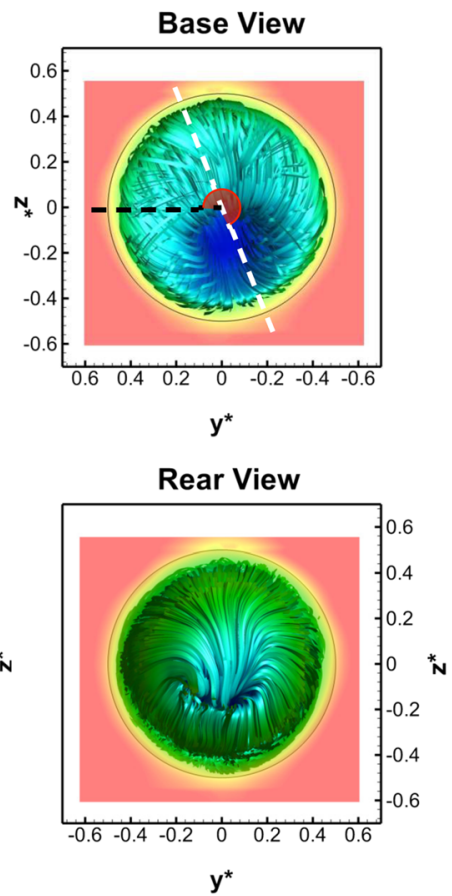

(d)

$u_{\text {LOM }}^{*}:-0.4 \quad-0.1 \quad 0.2 \quad 0.4 \quad 0.7 \quad 1.0$

FIG. 8. Temporal evolution of the wake recirculation obtained combining the time averaged field with the first two POD modes. First row: wake topology as seen from the model base, looking downstream; second row: wake topology as seen from a point downstream of the wake closure, looking upstream toward the base. The recirculating flow is visualised by means of stream ribbons coloured according to the non-dimensional values of the axial component of the velocity $u_{\text {LOM }}^{*}$ (for the sake of simplicity, ribbons with $u_{\text {LOM }}^{*}>0.5$ have been omitted). The white dashed line indicates the plane of reflectional symmetry. $t^{*}$ denotes the non-dimensional time $\left(\Delta t_{T P I V}^{*}=V_{\infty} /\left(D \cdot f_{T P I V}\right)\right)$. (a) $t^{*}=5 \cdot \Delta t_{T P I V}^{*}$ (b) $t^{*}=13 \cdot \Delta t_{T P I V}^{*}$ (c) $t^{*}=25 \cdot \Delta t_{T P I V}^{*}$ (d) $t^{*}=32 \cdot \Delta t_{T P I V}^{*}$

seen to appear and disappear simultaneously, as shown in Fig. 9 (Multimedia view). Additional coherent structures are also observed rolling up in the region of the shear layer. Close to the base $\left(x^{*} \leq 0.4\right)$, the distribution of such structures is not uniform because of the asymmetry in the wake topology. For $0.4<x^{*}<0.8$, however, a more even distribution of the same structures can be seen, arguably due to the occurrence of the shear layer instability documented in the work of Berger et al. ${ }^{5}$ and Duell and George; ${ }^{10}$ small longitudinal ribs (see the arrows in Fig. 9) connect the main transverse vortices, leading to the formation of a bigger toroidal structure (highlighted by dashed-dotted lines in Fig. 9). The four snapshots suggest that the overall topology of the torus does not undergo significant variations over time, although the small coherent structures are continuously shed and convected downstream. This is not the case for the hairpin vortex. The distribution of the structures forming such vortex indeed changes over time, as the wake switches from a state with just one plane of symmetry [dashed white line in Figs. 9(a), 9(c), and 9(d)] toward a more axisymmetric state [Fig. 9(b)]. In the snapshots reported in Fig. 9 (Multimedia view), it is also shown that the transition between consecutive reflectional symmetry preserving states tends to be a more "gradual" process (i.e., small changes around the initial position are more likely to occur than "phase" jumps) than that reported, for example, in the case of a bluff body with a rectangular base (Refs. 17 and 18). This is also documented in Fig. 9 (Multimedia view). The same trend is confirmed when considering the distribution of the changes in radial $\left(\Delta R^{*}{ }_{\mathrm{CoP}}\right)$ and azimuthal $\left(\Delta \theta_{\mathrm{CoP}}\right)$ positions of the centre of pressure over the model base between two consecutive time instants $\left(t^{*}\right.$ and $\left.t^{*}+\delta t^{*}\right)$, determined according to the equations

$$
\begin{gathered}
\Delta R_{\mathrm{CoP}}^{*}=R_{\mathrm{CoP}}^{*}\left(t^{*}+\delta t^{*}\right)-R_{\mathrm{CoP}}^{*}\left(t^{*}\right), \\
\Delta \theta_{\mathrm{CoP}}=\theta_{\mathrm{CoP}}\left(t^{*}+\delta t^{*}\right)-\theta_{\mathrm{CoP}}\left(t^{*}\right) .
\end{gathered}
$$

The results are presented in Fig. 10. An almost symmetric distribution around 0 is obtained for both $\Delta R^{*}{ }_{\mathrm{CoP}}$ and $\Delta \theta_{\mathrm{CoP}}$. This is consistent with the trend reported by Taneda ${ }^{41}$ when 


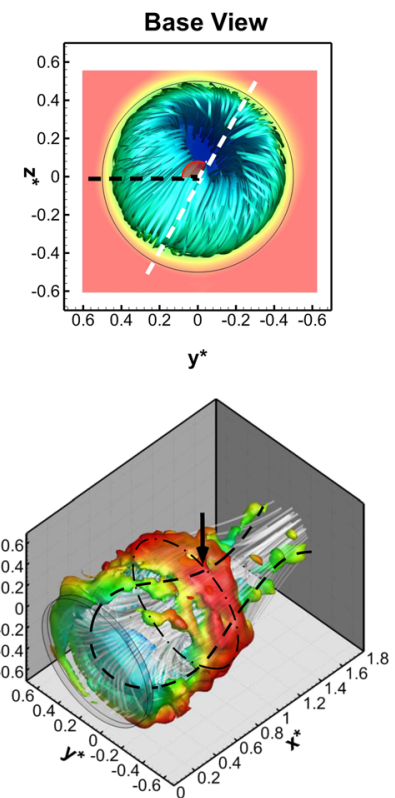

(a)

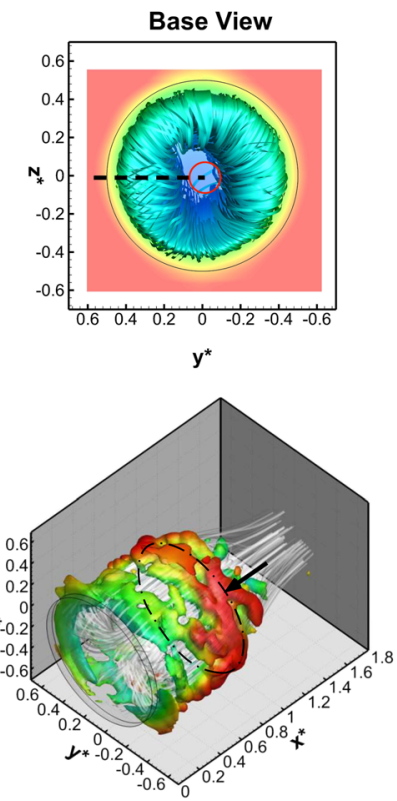

(b)
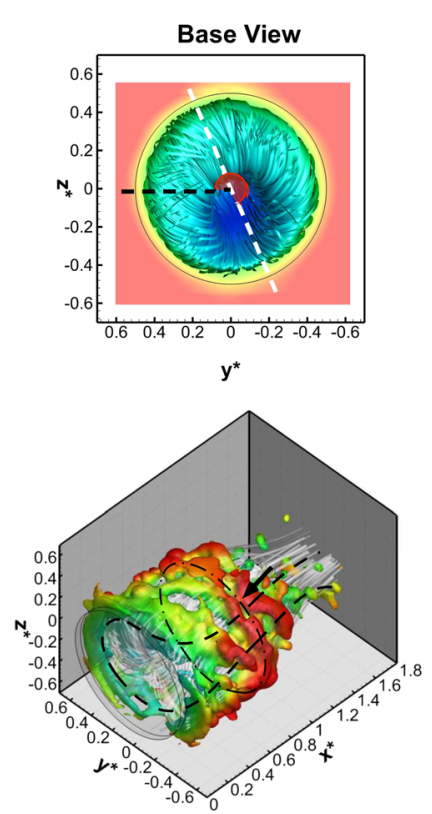

(c)

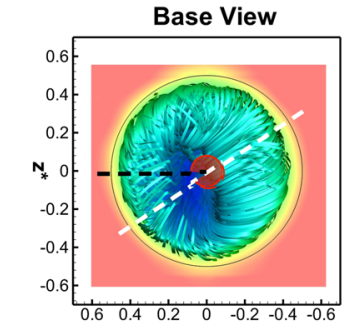

$y^{*}$

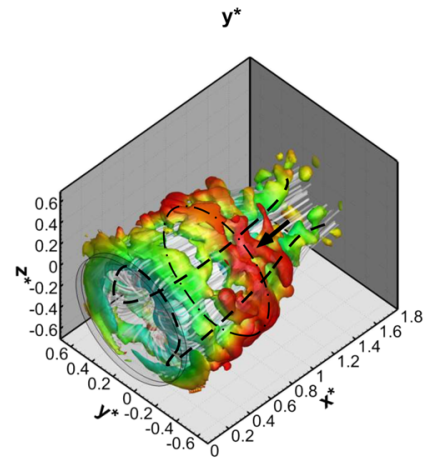

(d)

$$
u_{\text {LOM }}^{*}:-0.4 \quad-0.1 \quad 0.2 \quad 0.4 \quad 0.7 \quad 1.0
$$

FIG. 9. Temporal evolution of the wake's recirculation. Results obtained combining the time averaged field with the first two POD modes. In the top row, the recirculating flow is visualised by means of stream ribbons coloured according to the non-dimensional values of the axial component of the velocity $u_{L O M}^{*}$ (for the sake of simplicity, ribbons with $u_{\text {LOM }}^{*}>0.5$ have been omitted). The white dashed line indicates the plane of reflectional symmetry. In the bottom row, the recirculating flow is visualised by means of stream ribbons superimposed on iso-surfaces of $\lambda_{2}^{*}=-0.25$ coloured with the non-dimensional value of the axial component of the velocity $u_{\text {LOM. }}^{*} \cdot t^{*}$ denotes the non-dimensional time $\left(\Delta t_{T P I V}^{*}=V_{\infty} /\left(D \cdot f_{T P I V}\right)\right)$. (a) $t^{*}=20 \cdot \Delta t_{T P I V}^{*}$ (b) $t^{*}=21 \cdot \Delta t_{T P I V}^{*}$ (c) $t^{*}=22 \cdot \Delta t_{T P I V}^{*}$ (d) $t^{*}=23 \cdot \Delta t_{T P I V}^{*}$. Multimedia view: https://doi.org/10.1063/1.5078379.1

characterising the erratic motion of the wake developing downstream of a sphere.

The evolution of the coherent structures isolated in the near-wake region becomes clearer when the third and fourth POD modes are considered [see Figs. 7(c) and 7(d)]. As in the case of modes 1 and 2, these two modes are paired

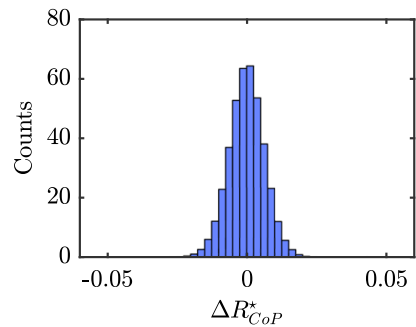

(a)

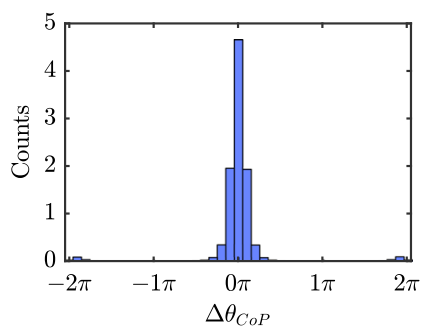

(b)
FIG. 10. Distributions of the changes in radial $\left[\Delta R_{C O P}^{*},(a)\right]$ and azimuthal $\left[\Delta \theta_{C O P}\right.$, (b)] positions of the centre of pressure between consecutive time instants $\left(t^{*}\right.$ and $\left.t^{*}+\delta t^{*}\right) \cdot t^{*}$ denotes the non-dimensional time $\left(t^{*}=V_{\infty} /\left(D \cdot f_{\text {samp }}\right)\right)$. Data recorded at $300 \mathrm{~Hz}$. since their energy level is similar and the spatial functions associated with them are in quadrature. The distribution of streamwise momentum associated with $\phi_{3_{\mathrm{V}}}$ and $\phi_{4_{\mathrm{V}}}$ shows an $m= \pm 1$ symmetry for $x^{*}<0.75$ and $x^{*}>0.75$. In contrast to that seen for the first pair of modes, however, the sign of $\phi_{n_{u^{*}}}$ is not constant across the length of the recirculating region. In fact, $\phi_{n_{u^{*}}}$ is observed to change sign between the two lobes at $x^{*} \approx 0.75$, resulting in a "helical" structure similar to the "Yin-Yang" mode observed in the numerical simulations performed by Yang et al. 46 when studying the turbulent wake behind a disk. Each lobe of the helix switches "side" while moving downstream of the base in the same way as the "tails" of the hairpin vortex characterising the RSP state are seen to bend. This structure is also reminiscent of the reflectional symmetry breaking (RSB) mode isolated in the laminar regime by Fabre et al. ${ }^{13}$ for the wake behind a circular disk. Furthermore, the location where the switch between the two lobes occurs is close to the location where Grandemange et al. ${ }^{20}$ placed the small control cylinder used for stabilising the wake, of a similarly shaped body, in an axisymmetric state.

The new pair of modes presented in Figs. 7(c) and 7(d) provides further proof of the existence of a dynamic link between 


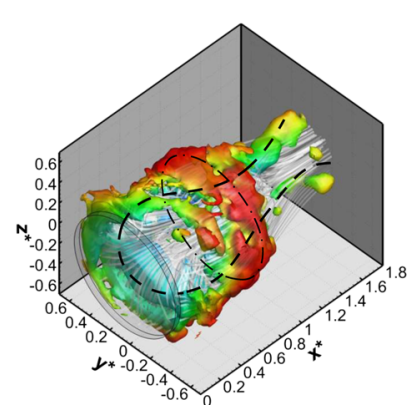

(a)

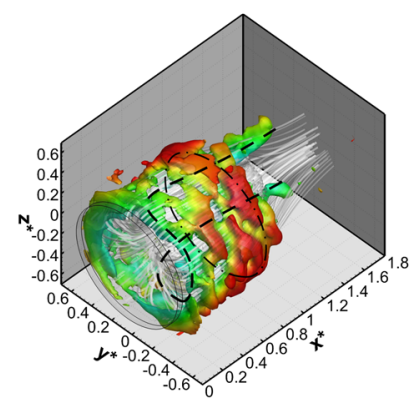

(e)

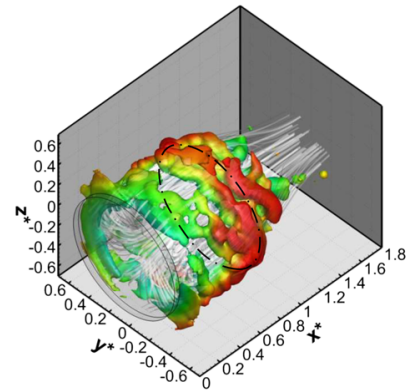

(b)

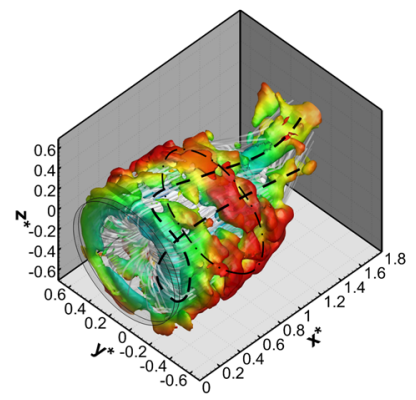

(f)

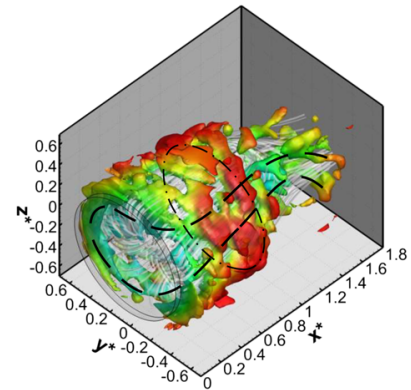

(c)

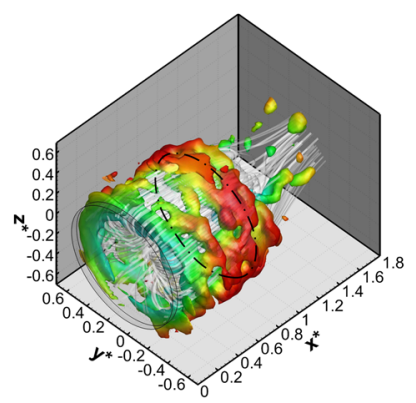

(g)

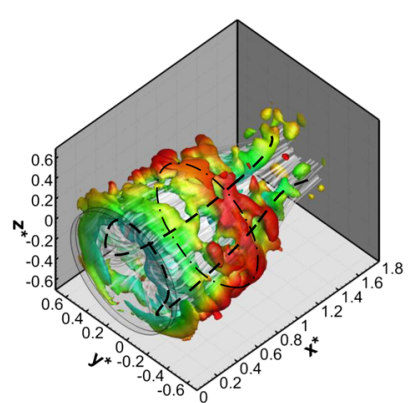

(d)

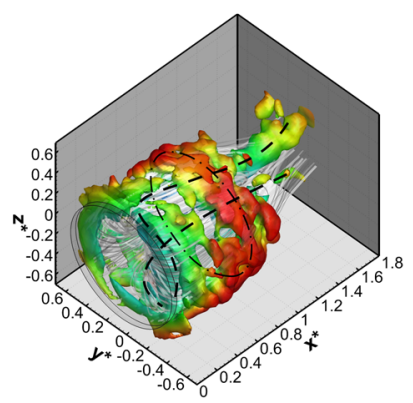

(h)

$$
\mathrm{u}_{\text {LOM: }}^{*} \quad-0.4 \quad-0.1 \quad 0.2 \quad 0.4 \quad 0.7 \quad 1.0
$$

FIG. 11. Temporal evolution of the wake's recirculation. Results obtained combining the time averaged field with the first four POD modes. The recirculating flow is visualised by means of stream ribbons superimposed on iso-surfaces of $\lambda_{2}^{*}=-0.25$ coloured with the non-dimensional value of the axial component of the velocity $u_{10 M}^{*} \cdot t^{*}$ denotes the non-dimensional time $\left(\Delta t_{T P I V}^{*}=V_{\infty} /\left(D \cdot f_{T P I V}\right)\right)$. (a) $t^{*}=20 \cdot \Delta t_{T P I V}^{*}$. (b) $t^{*}=21 \cdot \Delta t_{T P I V}^{*}$ (c) $t^{*}=22 \cdot \Delta t_{T P I V}^{*}$. (d) $t^{*}=23 \cdot \Delta t_{T P I V}^{*}$. (e) $t^{*}=24 \cdot \Delta t_{T P I V}^{*}$ (f) $t^{*}=25 \cdot \Delta t_{T P I V}^{*}(\mathrm{~g}) t^{*}=26 \cdot \Delta t_{T P I V}^{*}(\mathrm{~h}) t^{*}=27 \cdot \Delta t_{T P I V}^{*}$. Multimedia view: $h$ ttps://doi.org/10.1063/1.5078379.2

the "head" and the "tails" of the hairpin vortices, as highlighted by the results presented in Fig. 11 and its Multimedia view, which refer to a low order model built by adding this second pair of modes to the flow field already presented in Fig. 9 (Multimedia view). The "tails" of the hairpin vortices are now more clearly defined [Figs. 11(a), 11(c)-11(f), and 11(h)]. The formation of these structures indeed appears to be strictly linked to the development of the asymmetry in the azimuthal direction, as proven by the fact that the asymmetry itself is reduced when the "tails" weaken [Fig. 11(e)] or disappear [Figs. 11(b) and 11(g)]. On the other hand, when the azimuthal asymmetry reaches its maximum "strength," the longitudinal structures are seen to stretch downstream, as the transverse vortex expands toward the base [Figs. 11(a), 11(c), and 11(f)]. This process is similar to that described by Pavia et al. ${ }^{33}$ for a rectilinear three dimensional body with a bi-stable wake, confirming the existence of similar dynamics for turbulent wakes generated by simple three dimensional bluff bodies of different shapes. ${ }^{36}$ While stretching downstream, the "tails" do not run parallel to the body's axis of symmetry but instead bend toward the shear layer, in either the same direction [Figs. 11(c) and 11(f)] or opposite directions [Fig. 11(a)]. This results in a flapping motion of the wake closure, visible in Fig. 11 (Multimedia view).

More insights into the interactions between the hairpin vortex and the surrounding shear layer are provided by the higher order POD modes. Traces of $m=0$ and $m=2$ distributions, matching the third, fourth, and fifth POD pressure modes reported in Fig. 6, can be found in the higher order POD modes extracted from the TPIV dataset, with $n$ ranging from 5 to 10 (Fig. 12). In particular, $m=2$ distributions, with different azimuthal orientations, are obtained for the fifth [Fig. 12(a)], sixth [Fig. 12(b)], seventh [Fig. 12(c)], eighth [Fig. 12(d)], and tenth [Fig. 12(f)] modes, whereas a more axisymmetric shape, corresponding to an azimuthal wave number of $m=0$, is seen for the ninth mode [Fig. 12(e)]. These modes are reminiscent of those reported by Gentile et al. ${ }^{15}$ when analyzing time resolved PIV data acquired at different cross-planes downstream of the base of a similarly shaped body. However, they are characterised by a much lower energy content, arguably due to the more limited capability of TPIV to accurately resolve the shear layer bounding the wake. ${ }^{38}$ The $m=0$ distribution appears to be linked to the unsteady motion of the wake in the axial and radial directions, particularly evident in the 


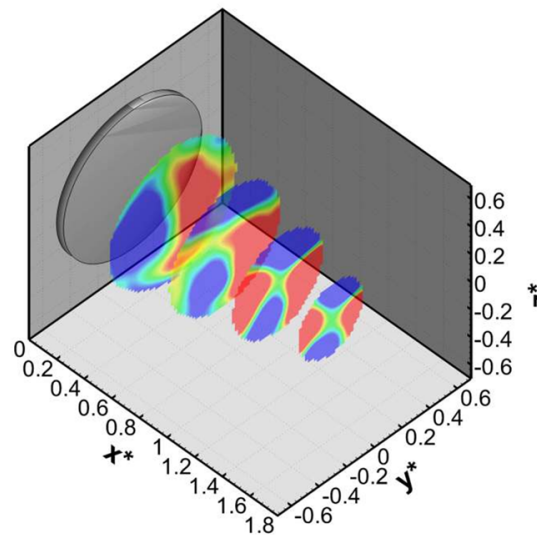

(a)

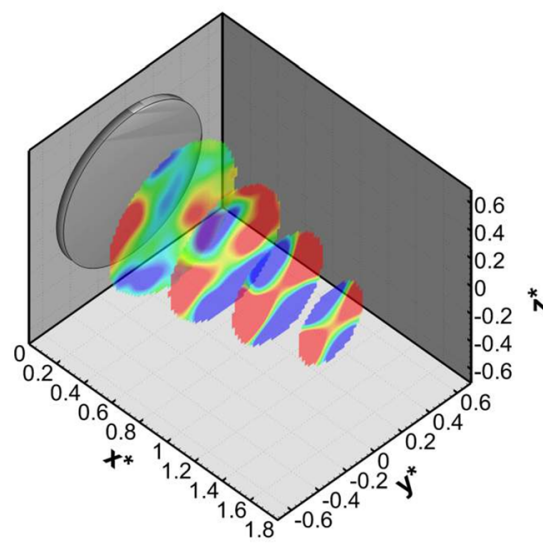

(d)

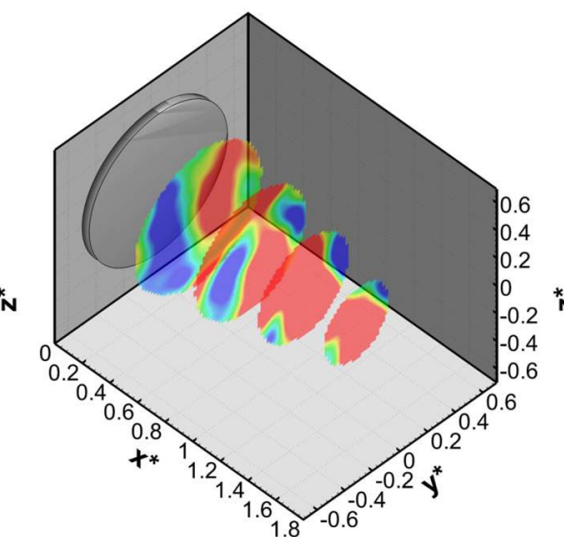

(b)

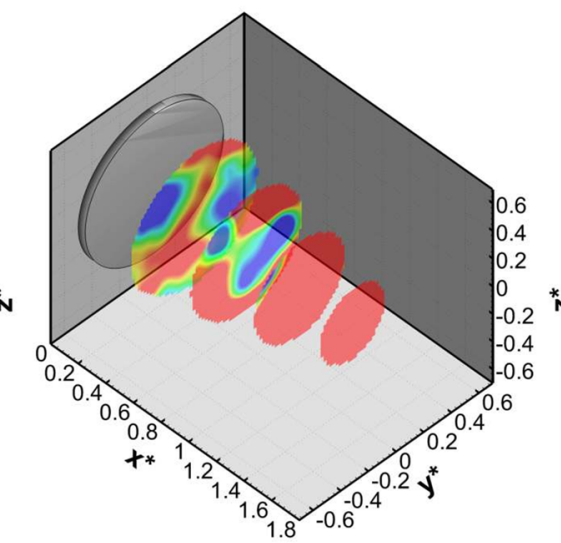

(e)

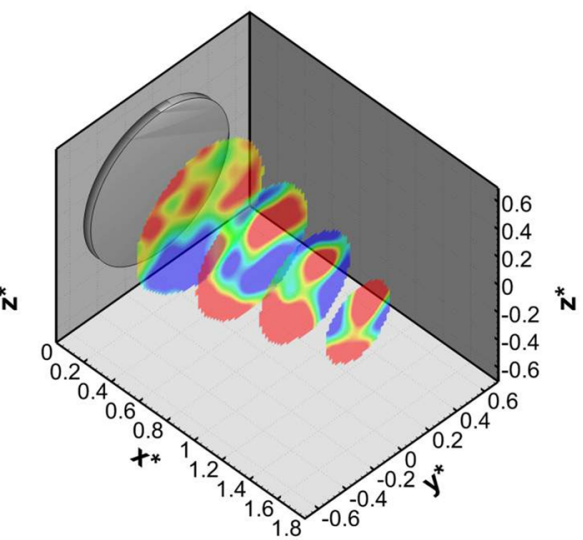

(c)

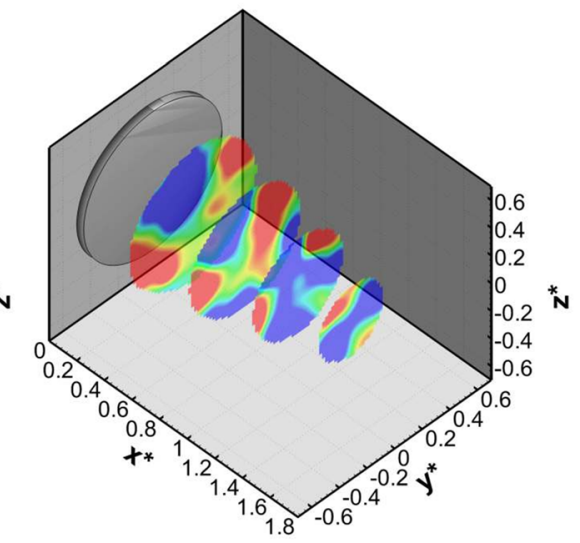

(f)

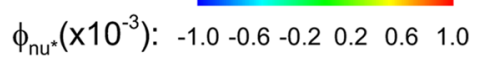

FIG. 12. Spatial functions of the POD modes extracted from the TPIV dataset $(5 \leq n \leq 10)$. The $2 \mathrm{D}$ slices are located at $x^{*}=0.375, x^{*}=0.75, x^{*}=1.125$, and $x^{*}=1.5$; they are coloured according to the values of the axial component of $\phi_{n_{\mathrm{V}^{*}}}$. Only the portion of the field of view with $\overline{u^{*}}<0.5$ has been considered for visualisation purposes. The energy level associated with each mode is: (a) $\phi_{5_{\mathrm{V}}}-1.68 \%$. (b) $\phi_{6_{\mathrm{V}}}-1.49 \%$. (c) $\phi_{7_{\mathrm{V}}}-1.34 \%$. (d) $\phi_{8_{\mathrm{V}}}-1.29 \%$. (e) $\phi_{9_{\mathrm{V}}}-1.17 \%$. (f) $\phi_{10_{\mathrm{V}}}-0.95 \%$.

region close to the base. In the same way, the $m=2$ shapes describe the evolution of the longitudinal "tails" of the hairpin structures.

When these modes are added to the low order model described in Eq. (14), the dynamic behavior previously discussed can still be recognised (see Fig. 13 and its Multimedia view) since the coherent structures forming the hairpin vortices are still visible. The "vortex tails" further increase in size [see the arrows in Figs. 13(a), 13(d), and 13(h)] and bend outwards even further, accentuating the "ovalisation" of the wake closure described in the work of Gentile et $a l .{ }^{15}$ As they interact with the shear layer, the "tails" burst into smaller vortical structures, clearly visible in Figs. 13(c) and 13(f) (see arrows). These structures are similar to those reported in the unsteady numerical simulations performed by Mariotti et al., ${ }^{29}$ for a similarly shaped body, and Evstafyeva, ${ }^{12}$ for geometries with a rectangular base. Their shedding has been found to be at the origin of the flapping motions of the wake closure that characterises the short-time wake dynamics. ${ }^{18,25}$

Additional information on such dynamics can be gathered from the spectral analysis of the signals recorded by pressure taps located at different radial locations. As shown in Fig. 14(a) for $\theta=\pi / 2 \mathrm{rad}$, a predominance of the wake pumping $\left(\mathrm{St}_{\mathrm{D}}=0.08\right)$ is observed in the region close to the centre of the base, in agreement with the trend reported in Fig. 12. A shift in frequency, from $\mathrm{St}_{\mathrm{D}}=0.08$ to $\mathrm{St}_{\mathrm{D}}=0.095$, is observed when moving towards the outer edge of the base. In the same 


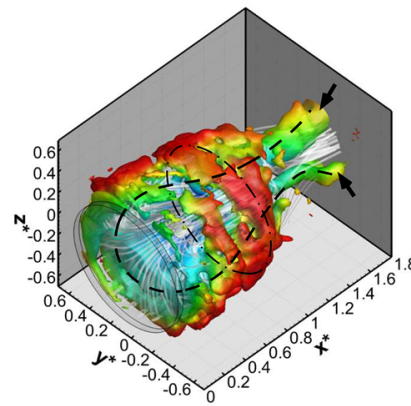

(a)

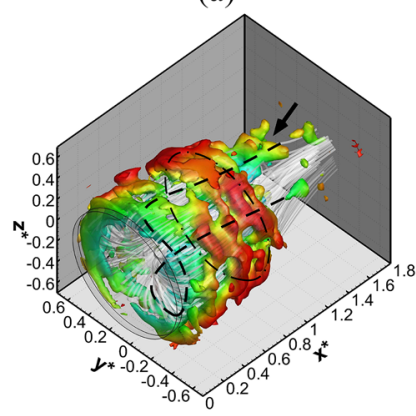

(e)

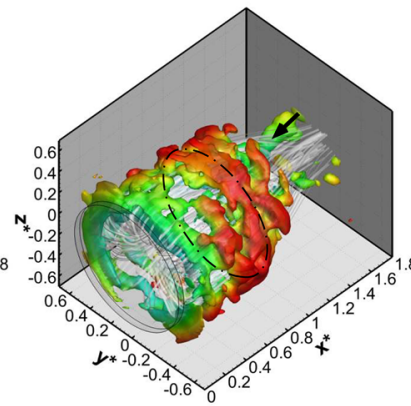

(b)

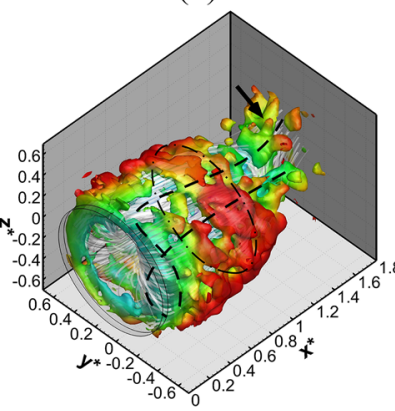

(f)

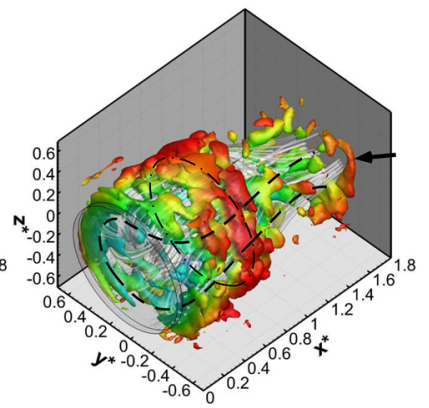

(c)

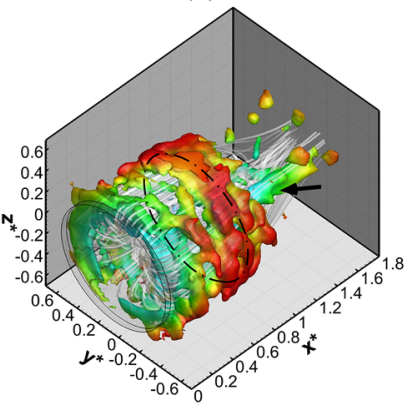

(g)

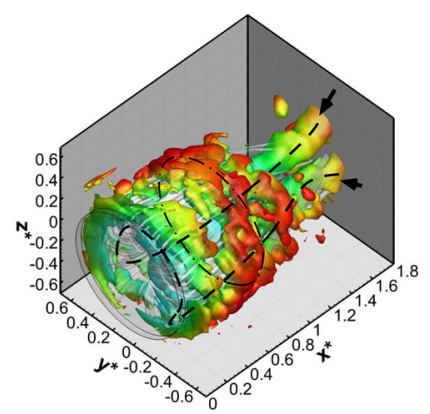

(d)

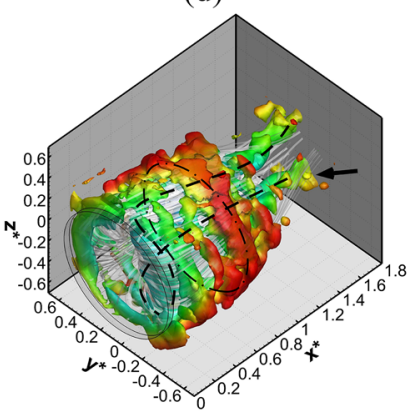

(h)

$u_{\text {LOM: }}^{*}:-0.4-0.10 .2 \quad 0.4 \quad 0.7 \quad 1.0$

FIG. 13. Temporal evolution of the wake's recirculation. Results obtained combining the time averaged field with the first ten POD modes. The recirculating flow is visualised by means of stream ribbons superimposed on iso-surfaces of $\lambda_{2}^{*}=-0.25$ coloured with the non-dimensional value of the axial component of the velocity $u_{\text {LOM }}^{*} \cdot t^{*}$ denotes the non-dimensional time $\left(\Delta t_{T P I V}^{*}=V_{\infty} /\left(D \cdot f_{T P I V}\right)\right)$. (a) $t^{*}=20 \cdot \Delta t_{T P I V}^{*}$ (b) $t^{*}=21 \cdot \Delta t_{T P I V}^{*}$ (c) $t^{*}=22 \cdot \Delta t_{T P I V}^{*}$ (d) $t^{*}=23 \cdot \Delta t_{T P I V}^{*}$ (e) $t^{*}=24 \cdot \Delta t_{T P I V}^{*}$ (f) $t^{*}=25 \cdot \Delta t_{T P I V}^{*}(\mathrm{~g}) t^{*}=26 \cdot \Delta t_{T P I V}^{*}(\mathrm{~h}) \mathrm{t}^{*}=27 \cdot \Delta \mathrm{t}_{\text {TPIV }}^{*}$. Multimedia view: $\mathrm{https}: / /$ doi.org/10.1063/1.5078379.3

conditions, the peak at $S t_{D}=0.235$, not visible for $\mathrm{R}^{*} \rightarrow 0$, also becomes evident.

Further information is added by the two-point coherence analysis performed considering taps placed at different locations. For example, when the sensors located, respectively, at $\left(R^{*}=0.429, \theta=\pi / 2 \mathrm{rad}\right)$ and $\left(R^{*}=0.429, \theta=3 / 2 \pi \mathrm{rad}\right)$ are considered [Fig. 14(e)], some level of coherence is seen only at $\mathrm{St}_{\mathrm{D}}=0.235$. Combined with the $\pi$ rad delay in the phase angle $\chi$, this further confirms the flapping nature of the motion associated with this peak. No coherence is observed at either $\mathrm{St}_{\mathrm{D}}=0.08$ or $\mathrm{St}_{\mathrm{D}}=0.095$, but a peak at $\mathrm{St}_{\mathrm{D}}=0.08$ is seen when the same analysis is performed considering one of the pressure sensors on the outermost ring (at $\left.R^{*}=0.429\right)$ and the tap located at the centre of the model base [at $R^{*}=0$, as shown in Fig. 14(f)]. A phase angle of 0 rad is observed in this case, confirming the pulsating nature of this mode. The same value of $\chi$ is observed for the peak at $\mathrm{St}_{\mathrm{D}}=0.095$, obtained when calculating the coherence between two taps located, respectively, at $R^{*}=0.429$ and $R^{*}=0.286$, having fixed for simplicity the azimuthal position at $\theta=\pi / 2 \mathrm{rad}$ [Fig. 14(g)]. A second peak at $S t_{D}=0.235$ is also visible in this case, in agreement with that seen when considering the auto-spectra of the signals recorded by the taps located close to the outer edge of the base.
These trends are confirmed when the coherence analysis is extended to all the taps located on the base, having selected data recorded by one of the taps located on the outermost ring (at $R^{*}=0.429$ ) as the first element of Eqs. (10) and (11) and fixed the frequency at either $\mathrm{St}_{\mathrm{D}}=0.08$ [Fig. 14(b)], $\mathrm{St}_{\mathrm{D}}=0.095$ [Fig. 14(c)], or $\mathrm{St}_{\mathrm{D}}=0.235$ [Fig. 14(d)]. Similarities are seen in the phase maps obtained for $S t_{D}=0.08$ and $S t_{D}=0.095$. In both cases, the regions with the highest level of coherence are in phase with the signal recorded by using the selected sensor. The main difference between these two cases is in the size of the region with $\Lambda>0.075$. This region is confined to the outer edge of the base for $\mathrm{St}_{\mathrm{D}}=0.095$, whereas it appears to extend toward the centre for $\mathrm{St}_{\mathrm{D}}=0.08$. This is different from that seen for $S t_{D}=0.235$, for which a region of coherence and $\chi=\pi \mathrm{rad}$ is observed only at $\theta=\pi \mathrm{rad}$ from the location of the reference tap, whereas no coherence is seen with the signals recorded in proximity to the centre of the base. The fact that the peak at $\mathrm{St}_{\mathrm{D}}=0.235$ is only visible close to the outermost edge of the base is consistent with the fact that this frequency is related to unsteady motions located mostly in proximity to the wake closure. ${ }^{28}$

Together these data allow a more accurate model of the short-time unsteady evolution of the near wake to be described. The modes at $\mathrm{St}_{\mathrm{D}}=0.08$ and $\mathrm{St}_{\mathrm{D}}=0.095$ appear to 


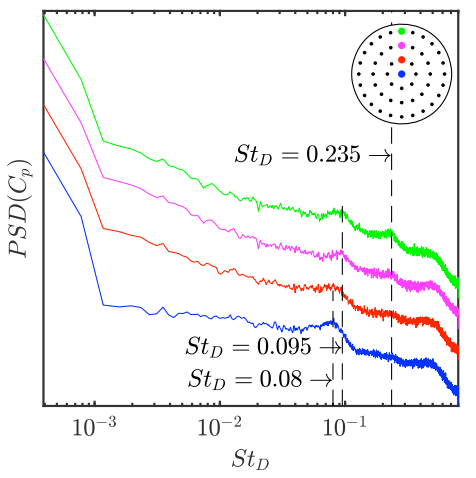

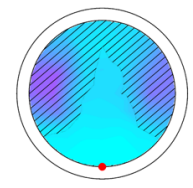

(b)

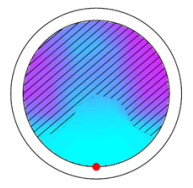

(c)

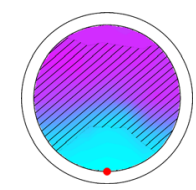

(d) (a)

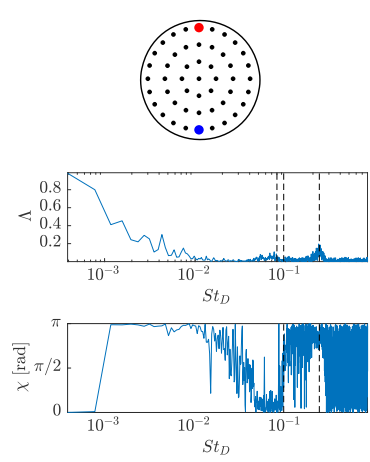

(e)

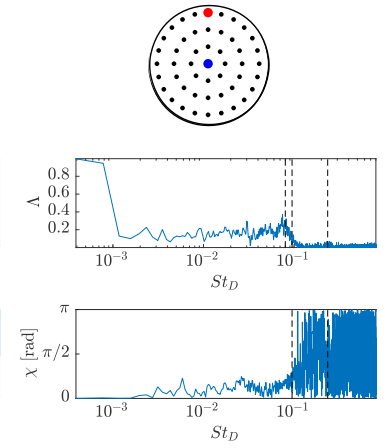

(f)

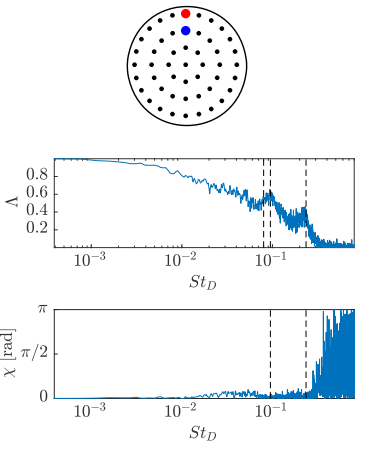

(g) be complementary, as they are both associated with the alternate expansion and contraction of the hairpin vortices isolated in Figs. 9, 11, and 13 (Multimedia view), with the first frequency prevailing at $\mathrm{R}^{*} \approx 0$, where the transverse vortex developing close to the base (the "head" of the hairpin vortex) is seen to continuously "stretch" and "squeeze," 33 and the second frequency becoming visible close to the outer edge of the base, where the interactions between the tails of the same vortices and the shear layer become predominant. As a result of such interactions, the tails "burst" into smaller vortical structures, ${ }^{25}$ whose shedding is at the origin of the peak at $\mathrm{St}_{\mathrm{D}}=0.235$ isolated in the pressure dataset, close to the model trailing edge. Time resolved TPIV data, however, are needed to conclusively prove this thesis.

\section{Effects of the wake dynamics on the base drag}

The existence of a relationship between the instantaneous base pressure distribution and the unsteady evolution of the wake has been investigated by considering the joint probability density functions of the value of the base drag $C_{D_{\text {Base }}}$ recorded at any time instant $t^{*}$ and the radial $R_{C o P}^{*}$ and azimuthal $\theta_{\text {CoP }}$ positions of the centre of pressure, determined from the base pressure data according to Eq. (3). The results for the original dataset sampled at $300 \mathrm{~Hz}$ are presented in Figs. 15(a) and 15(d).

An almost circular distribution is obtained in the plane $\mathrm{C}_{\mathrm{D}_{\text {Base }}}, \mathrm{R}_{\mathrm{CoP}}^{*}$ [Fig. 15(a)], as the points tend to cluster around the position $R_{\mathrm{CoP}}^{*} \approx 0.025, C_{\mathrm{D}_{\text {Base }}} \approx 0.190$ (the fact that $\mathrm{R}_{\mathrm{CoP}}^{*} \neq 0$ for most of the time confirms the non-axisymmetric nature of the pressure distribution over the model base, at least from an unsteady point of view). At the same time, all azimuthal positions are equally explored [Fig. 15(d)], with a slight predominance of the position at $\theta_{\mathrm{CoP}} \approx 3 / 4 \pi$, arguably due to the presence of some small residual asymmetries in the experimental setup, which may also explain the slight eccentricity observed in the time averaged base pressure map reported in Fig. 4(a).

An interesting trend emerges when the bin-average of the values of $\mathrm{C}_{\mathrm{D}_{\text {Base }}}$ recorded at different radial positions is performed [red curve in Fig. 15(a)]. In this case, bins of $1.0 \times 10^{4}$ data points (equal to $5.6 \%$ of the length of the original dataset) have been considered. As can be seen in Fig. $15(\mathrm{a}), \widetilde{\mathrm{C}}_{\mathrm{D}_{\text {Base }}}$ tends to drop from the time averaged value $\left(\bar{C}_{D_{\text {Base }}}=0.189\right)$ when either $R_{\mathrm{CoP}}^{*}<0.03$ or $R_{\mathrm{CoP}}^{*}>0.03$. This seems to suggest the existence of two different low-drag scenarios. The first state, with $R_{\mathrm{CoP}}^{*} \rightarrow 0$, is characterised by the restoration of the symmetry of the wake in the axial direction [as seen, for example, in Figs. 11(b) and 11(c)] and is consistent with the results reported in the work of Grandemange et al. ${ }^{20}$ for a similar case. The second state, with $R_{C o P}^{*} \rightarrow 0.04$, corresponds to the selection of one of the reflectional symmetry preserving states shown in Figs. 8, 9, and 11 (Multimedia view) and seems to confirm the trend reported by Oxlade ${ }^{31}$ when controlling the wake past a similarly shaped body using a high frequency pulsed jet located close to the model trailing edge. 


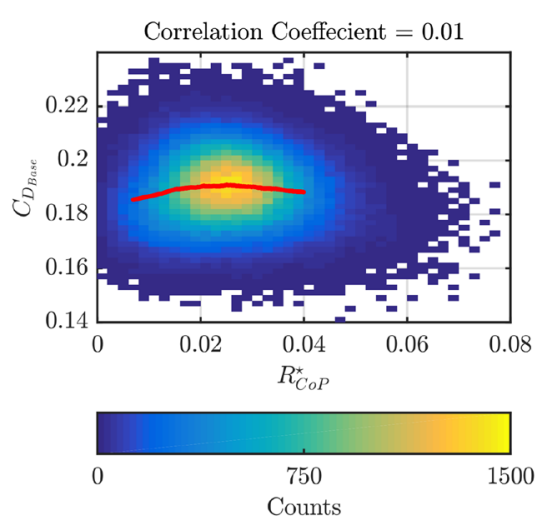

(a)

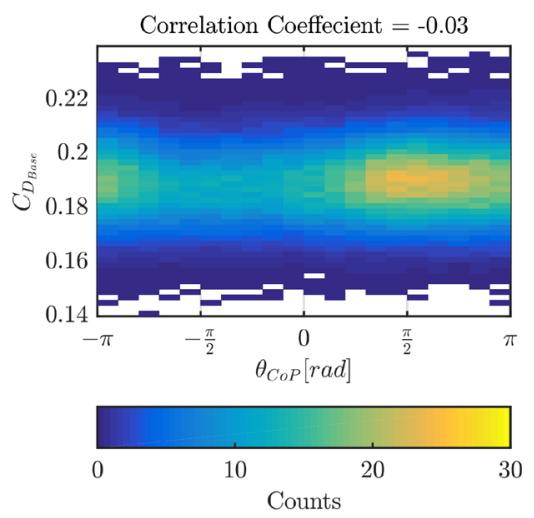

(d)

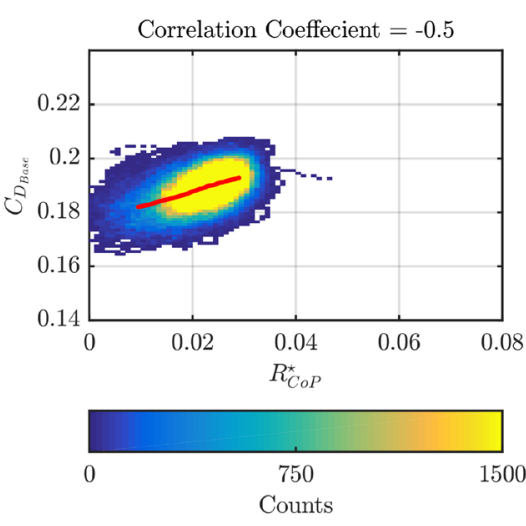

(b)

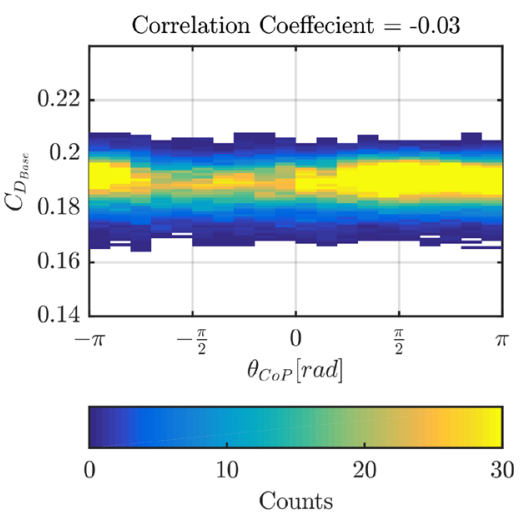

(e)

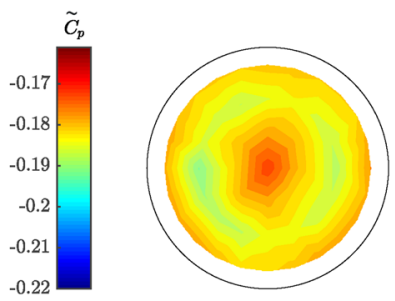

(g)

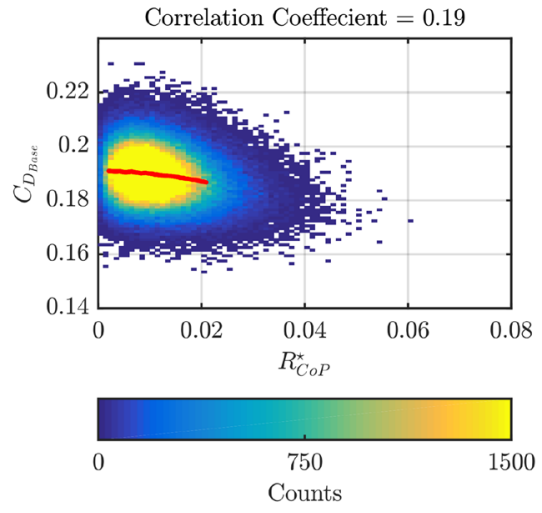

(c)

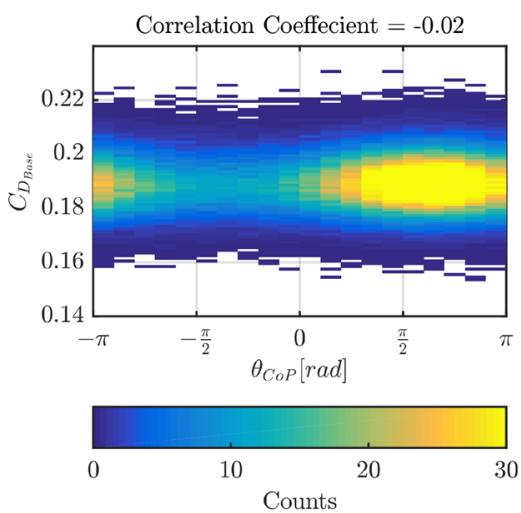

(f)

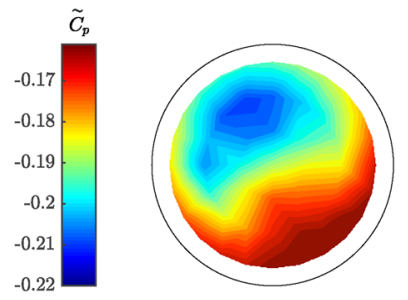

(h)

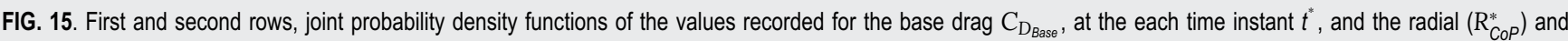
azimuthal $\left(\theta_{C O P}\right)$ positions of the centre of pressure at the same instant: [(a) and (d)] data recorded in the experiment at $300 \mathrm{~Hz}$; [(b) and (e)] data filtered with a low-pass filter; [(c) and (f)] data filtered with a high-pass filter. In both filtered cases, the cut-off frequency is at $5 \mathrm{~Hz}$. The red curves refer to the values of $\mathrm{C}_{\mathrm{D}_{\text {Base }}}$ averaged in bins of $1.0 \times 10^{4}$ data points (equal to $5.6 \%$ of the length of the original dataset). [(g) and $\left.(\mathrm{h})\right]$ Base pressure distributions obtained for the low-drag case scenarios isolated from the low-pass and high-pass filtered datasets. The conditional average has been performed considering the data points associated with the smallest $1 \%$ and the largest $1 \%$ of the values of $R_{C O P}^{*}$.

Only one of the two low-drag configurations is preserved when the data are filtered using either a low-pass or a highpass filter, having applied a cut-off frequency of $5 \mathrm{~Hz}$ (or $\mathrm{St}_{\mathrm{D}}$ $=0.027$ ) in both cases. This frequency is higher than the frequency characteristic of the long-time instability but lower than any of the frequencies characterising the short-time wake dynamics discussed in Sec. III B 1.

As can be seen in Fig. 15(b), when the data are low-pass filtered, only the left branch of the curve reported in Fig. 15(a) is kept. Therefore, the only low-drag configuration admissible is the axisymmetric one (with $R_{\mathrm{CoP}}^{*} \rightarrow 0$ ). Furthermore, the fact that in this case the values of $\mathrm{R}_{\mathrm{CoP}}^{*}$ are not seen to increase beyond $R_{\mathrm{CoP}}^{*} \approx 0.03$ seems to suggest that higher deviations in the radial position of the centre of pressure are a consequence of the short-time wake dynamics. The very low frequency motion, on the other hand, is still clearly visible, as highlighted by the uniform distribution observed for the joint probability density function obtained for $C_{D_{\text {Base }}}$ and $\theta_{\text {CoP }}$ 
[Fig. 15(e)]. As expected, the contribution of such a mode to the overall wake dynamics is now even stronger than before. This becomes evident when POD is applied to the low-pass filtered data. As shown in Fig. 16, although no major changes are reported for the shapes of the spatial modes between this case and the unfiltered dataset discussed in Sec. III B 1, a significant variation is seen in the energy content of the first two modes, which is observed to almost double up. The most significant change in the shape of the spatial functions obtained in the two cases is in the third mode. The $m=0$ distribution seen in Fig. 6(c) for the unfiltered case is replaced by a more "elongated" shape, with just two planes of symmetry. This shape is not dissimilar from that reported by Pavia et al. ${ }^{33}$ when testing a simplified square-back automotive shape at a yaw angle of $\Psi \approx 0^{\circ}$. In that case, a relationship was found between this mode, associated with the motion of the wake mostly in the axial direction, and the lateral symmetry breaking bi-stable mode first described in the work of Grandemange et al. ${ }^{18}$ In particular, it was shown that the temporal coefficient associated with such a mode tends to reach higher values when the temporal coefficient linked to the lateral symmetry breaking mode goes to zero, suggesting that an increase in base pressure occurs every time the lateral symmetry of the wake is restored. A similar trend is seen for the axisymmetric body of the present investigation, when a low-pass filter is applied to the original dataset [Fig. 18(e)]. An indication of this relationship is visible in the joint probability density functions of $a_{3}$ and $a_{1}$, obtained for the unfiltered signal. However, it becomes much clearer when a low-pass filter is applied to the same data [Fig. 18(e)]. This trend is consistent with that shown by the low-order unsteady velocity fields presented in Figs. 9 and 11 (Multimedia view). When the wake recovers its axial symmetry and the hairpin vortex observed in the reflectional symmetry preserving states disappears, the only large coherent structure that is left is the ring vortex shown in Figs. 9(b), 11(b), and 11(g) (Multimedia view). This structure is located further downstream from the base than the "head" of the hairpin vortex seen in the RSP states. Its interaction with the model base is therefore weaker, allowing a better pressure recovery, especially in proximity to the centre of the base, where the amount of reverse flow increases. The existence of such a state is confirmed by the base pressure distribution obtained when averaging the pressure fields associated with the smallest $1 \%$ of the values of $R_{C o P}^{*}$. As shown in Fig. $15(\mathrm{~g})$, the axisymmetric distribution resulting from this conditional average is rather different from that produced by the temporal average reported in Fig. 4(a) or in the work of Rigas et al. ${ }^{36}$ It is characterised by the presence of an annular low pressure region developing at $\mathrm{R}^{*} \approx 0.5$ and surrounded by a ring of higher pressure located in proximity to the trailing edge of the model. Unlike that seen in previous cases [Fig. 4(a) and Ref. 36] however, the latter region is no longer the portion of the base characterised by the highest values of $C_{p}$, as this appellative now belongs to the centre of the base itself. A similar distribution was reported in the work of Pavia et al. ${ }^{33}$ for the symmetry preserving state of the wake of a rectilinear three-dimensional model with a rectangular base, further confirming the similarities between these two cases. $A \approx 4.2 \%$ drop in $C_{\text {Base }}$ over the time averaged value discussed in Sec. III A is seen in these conditions (from $\mathrm{C}_{\mathrm{Base}}=0.189$ to $\widetilde{\mathrm{C}}_{\mathrm{Base}}=0.181$ ). This is close to the gain reported by Grandemange et al. ${ }^{20}$ when forcing the axial symmetry of the wake of a similarly shaped body.

The second low drag scenario visible in Fig. 15(a), for $\mathrm{R}_{\mathrm{CoP}}^{*}>0.03$, is of a different nature. This is the only lowdrag state that is still visible in the joint probability density function distribution of $\mathrm{C}_{\mathrm{D}_{\text {Base }}}$ and $\mathrm{R}_{\mathrm{CoP}}^{*}$, when the high-pass filtered dataset is considered [Fig. 15(c)]. In fact, $R_{\mathrm{CoP}}^{*} \rightarrow 0$ represent in this case the condition associated with the highest values of $\widetilde{C}_{B a s e}$. The base drag, on the other hand, is seen to decrease as $R_{\text {CoP }}^{*} \rightarrow 0.04$, although the trend is much weaker than that observed for the low-pass filtered signal, as highlighted by the noticeable reduction observed in the magnitude of the correlation coefficient between the two cases [from 0.5 of Fig. 15(b) to 0.19 of Fig. 15(c)]. In such conditions, the wake tends to lock in a symmetry preserving state, following the stabilisation of the hairpin vortex structure described in

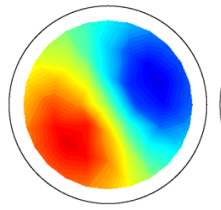

(a)

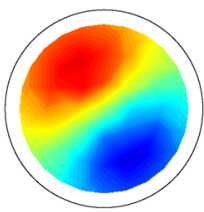

(b)

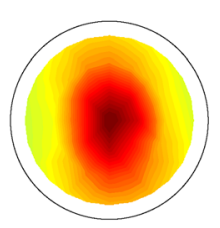

(c)

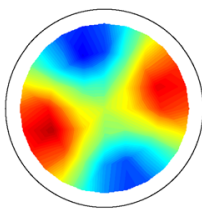

(d)

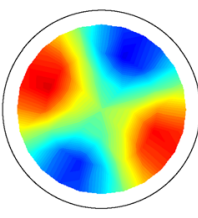

(e)

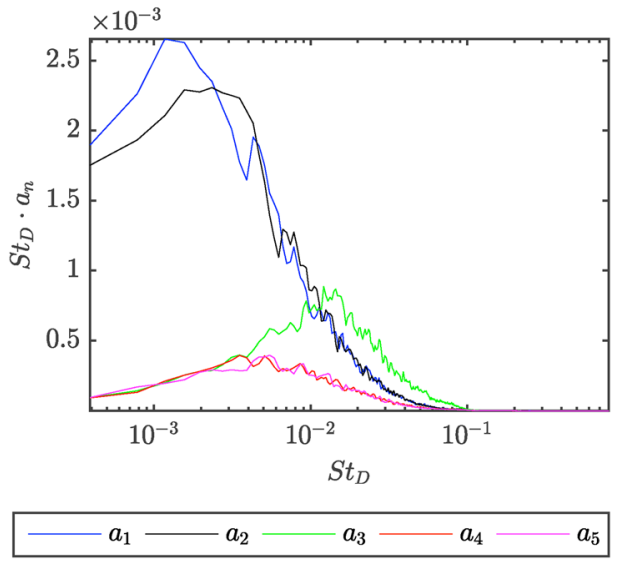

(f)

)
FIG. 16. [(a)-(e)] Spatial functions associated with the first five POD modes extracted from the base pressure data filtered with a low-pass filter. In each figure, the continuous line indicates the location of the model trailing edge. (f) Premultiplied spectra of the temporal coefficients associated with the same modes. The energy content associated with each mode is: (a) $\phi_{1_{C p}}-39.8 \%$. (b) $\phi_{2_{\mathrm{Cp}}}-37.8 \%$. (c) $\phi_{3_{\mathrm{Cp}}}-8.5 \%$. (d) $\phi_{4_{\mathrm{Cp}}}-4.7 \%$. (e) $\phi_{5_{\mathrm{Cp}}}-4.6 \%$. 


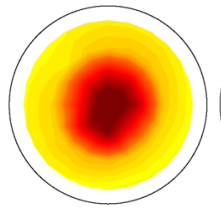

(a)

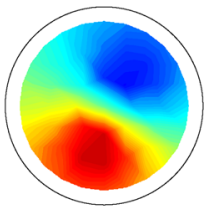

(b)

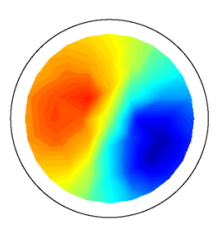

(c)

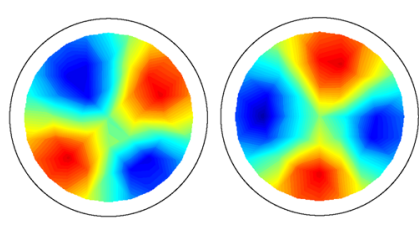

(d)

(e)

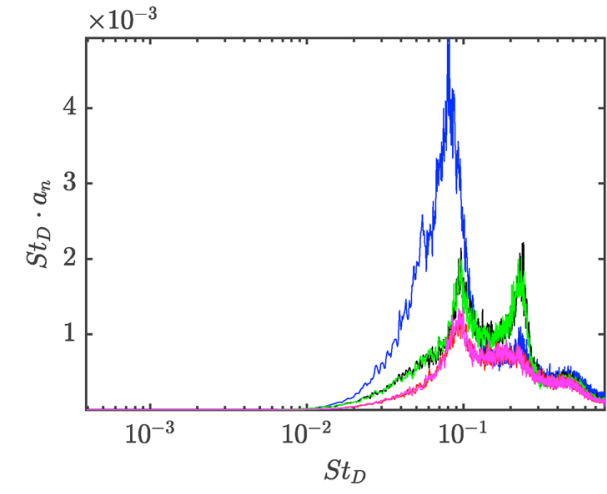

$-a_{1}-a_{2}-a_{3}-a_{4}-a_{5}$

(f)

FIG. 17. [(a)-(e)] Spatial functions associated with the first five POD modes extracted from the base pressure data filtered with a high-pass filter. In each figure, the continuous line indicates the location of the model trailing edge. ( $f$ ) Premultiplied spectra of the temporal coefficients associated with the same modes. The energy content associated with each mode is: (a) $\phi_{1_{\mathrm{Cp}}}-14.9 \%$. (b) $\phi_{2_{C p}}-9.1 \%$. (c) $\phi_{3_{C p}}-8.9 \%$. (d) $\phi_{4_{\mathrm{Cp}}}-5.9 \%$. (e) $\phi_{5_{\mathrm{C} p}}-5.9 \%$.
Sec. III B 1 . In particular, the position around $\theta_{\mathrm{CoP}} \approx 3 / 4 \pi$ is selected, as suggested by the distribution obtained in this case for the joint probability density function of $\mathrm{C}_{\mathrm{D}_{\text {Base }}}$ and $\theta_{\mathrm{CoP}}$ [Fig. 15(f)]. This position already appears to be a weak attractor when the full dataset is considered [Fig. 15(d)], arguably as a result of the presence of a (small) residual asymmetry in the experimental setup. Its selection is a direct consequence of the suppression of the very low frequency dynamics. The lowdrag state obtained in this case is characterised by a much stronger pressure gradient in the direction of the plane of reflectional symmetry compared to that in the radial direction for the low-pass filtered data [Fig. 15(g)], as two regions with, respectively, lower and higher pressure than the lowand high-pressure regions observed in Fig. 15(g) coexist. This is evident in the base pressure distribution obtained, performing the average of the pressure fields associated with the highest $1 \%$ of the values of $\mathrm{R}_{\mathrm{CoP}}^{*}$ [Fig. 15(h)]. Nevertheless, the resulting value of the base drag is only $\approx 0.001$ higher than that obtained for the axisymmetric distribution previously discussed.

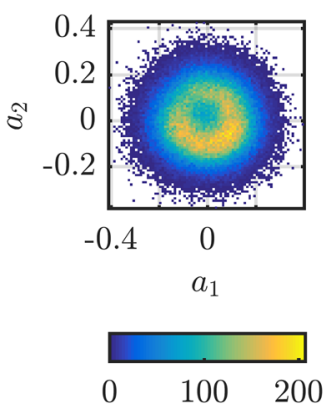

(a)

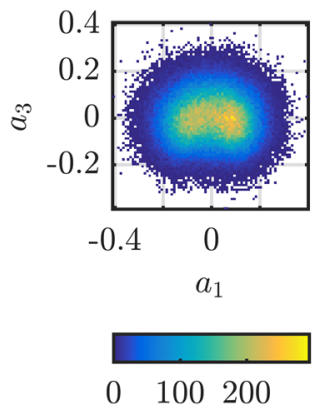

(d)

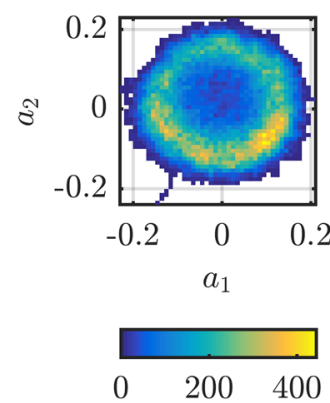

(b)

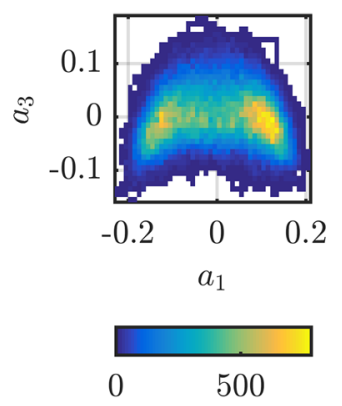

(e)

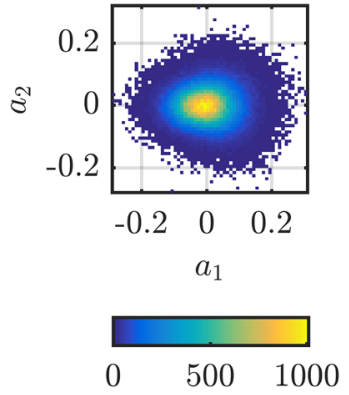

(c)

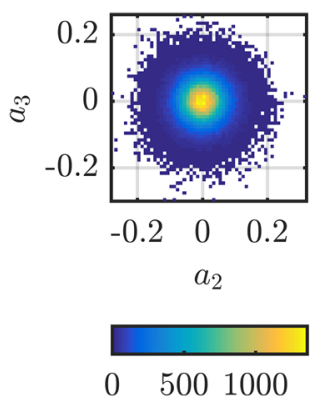

(f)
FIG. 18. Joint probability density functions of the second and first POD temporal coefficients [(a)-(c)], third and first POD temporal coefficients [(d) and (e)], and third and second POD temporal coefficients (f) obtained for [(a) and (d)] data recorded in the experiment at $300 \mathrm{~Hz}$; [(b) and (e)] data filtered with a low-pass filter; $[(c)$ and $(f)]$ data filtered with a high-pass filter. In both filtered cases, the cut-off frequency is at $5 \mathrm{~Hz}$. 
The effects of the high pass filter become even more visible when POD is applied to the same dataset. Having filtered out the very low frequency motions changes the ranking of the different POD modes quite noticeably. As can be seen in Fig. 17(e), a direct consequence of the stabilisation of the wake around a single plane of reflectional symmetry is the reduction in the energy content of the modes featuring an azimuthal wave number of $m= \pm 1$. The energy level of such modes drops by more than $100 \%$ compared to that in the unfiltered dataset reported in Fig. 6, as the only coherent motions that these modes are now able to capture are those associated with the frequency peaks at $\mathrm{St}_{\mathrm{D}}=0.095$ and $\mathrm{St}_{\mathrm{D}}=0.235$ [Fig. 17(f)]. The energy content of these modes is now lower than that associated with the $m=0$ mode, arguably because close to the base the "wake pumping" at $\mathrm{St}_{\mathrm{D}}=0.08$ is stronger than the motions at $\mathrm{St}_{\mathrm{D}}=0.095$ and $\mathrm{St}_{\mathrm{D}}=0.235$, as the latter modes stem from the interactions between the hairpin vortex and the vortex ring developing in correspondence of the wake closure, as explained in Sec. III B 1. Unlike the low-pass case, however, no relationship is found when looking at the joint probability density function distribution between the temporal coefficients related to the modes with $m=0$ and $m= \pm 1$ [Fig. 18(c)]. Similar conclusions can be extended to the plot showing the joint probability density function distribution between $a_{2}(t)$ and $a_{3}(\mathrm{t})$. Unlike that seen in Figs. 18(a) and 18(b), where the values of the temporal coefficients obtained for the $m= \pm 1$ modes tend to arrange in an annular pattern as a consequence of the long time erratic motion of the flow reversal, ${ }^{15}$ no correlation is visible in the high-pass filtered dataset between the temporal coefficients associated with such modes [Fig. 18(f)].

\section{SUMMARY AND CONCLUSIONS}

The near-wake of an axisymmetric body with a lengthto-diameter ratio $L / D=5$ has been studied using an array of base pressure tappings and large scale Tomographic Particle Image Velocimetry (TPIV), at a diameter based Reynolds number of $R e_{\mathrm{D}}=3.2 \times 10^{5}$. Base pressure measurements have shown a good match with the existing literature. ${ }^{36}$ The application of Proper Orthogonal Decomposition (POD) to the pressure data has confirmed the existence of three different coherent motions of the wake: a very low frequency mode, resulting in a long-time erratic motion of the wake similar to that described by Taneda ${ }^{41}$ for a sphere; a flapping motion of the shear layer, ${ }^{20}$ occurring at a normalised frequency of $\mathrm{St}_{\mathrm{D}} \approx 0.23$; and a pumping motion. ${ }^{5}$

Further insights into the near-wake topology and its dynamics have been extracted from the application of snapshot POD to the TPIV data. The first pair of modes has the same $m= \pm 1$ symmetry seen in the corresponding modes extracted from the base pressure field. From the low order model built combining the time averaged field with these two modes, it can be inferred that the axisymmetric topology seen for the time averaged wake is the result of the succession of different Reflectional Symmetry Preserving (RSP) states, each one featuring a single plane of symmetry randomly oriented in the azimuthal direction. The RSP states are characterised by the presence of a transverse vortex developing close to the model base and two streamwise vortical structures forming downstream of the wake closure. These structures are dynamically linked together, resulting in a "hairpin vortex."

The hairpin vortices are randomly shed from different azimuthal locations, although some level of axial symmetry is recovered in the shear layer forming in proximity to the wake closure (for $0.4<x^{*}<0.8$ ). Remarkable similarities are found with that described in the work of Pavia et al. ${ }^{33}$ for the wake of a square-back body in the ground effect, suggesting the existence of a "universal" dynamic behavior for the wakes of three-dimensional bluff bodies. ${ }^{36}$

Further proof of the existence of a "dynamic link" between the "head" and the "tails" of the hairpin vortex seen in the RSP states is provided by a second pair of POD modes, not visible in the pressure dataset. These modes are characterised by a twisted two-lobe structure, reminiscent of the reflectional symmetry breaking mode isolated in the laminar regime by Fabre et al. ${ }^{13}$ They describe the contraction/expansion process experienced by the hairpin vortices. As the "head" of the hairpin vortex stretches downstream, its "tails" are seen to deviate from the body's axis, bending toward the shear layer. After having reached a maximum, the hairpin vortex starts to shrink, until the axial symmetry is recovered (at least in part). The process then starts again, with a new hairpin vortex developing in either a similar or different azimuthal location, depending on any random perturbation.

The analysis of the temporal evolution of the radial position of the centre of pressure $R_{C o P}^{*}$ over the model base has demonstrated the existence of two low-drag scenarios. The first one, obtained when $R_{\text {CoP }}^{*} \rightarrow 0$, is characterised by the restoration of the symmetry of the wake in the axial direction, arguably as a consequence of the disappearance of the hairpin vortex. The second state, with $R_{\mathrm{CoP}}^{*} \rightarrow 0.04$, corresponds to the selection of a single reflectional symmetry preserving state, whose orientation appears to be dictated by any small asymmetry present in the experimental setup Oxlade. ${ }^{31}$ The fact that only one of these two states remains when the data are filtered with either a low-pass filter or a high-pass filter (both with a cut-off frequency of $5 \mathrm{~Hz}$ ) provides useful information for the implementation of flow control strategies aiming to reduce the drag. It is shown that the selection of an axisymmetric pressure distribution is not a necessary condition to achieve a base drag reduction, although it is the only admissible low-drag scenario when the short-time wake dynamics are filtered-out, for example, by adding a cavity to the model base, as shown by Lucas et al. ${ }^{25}$

The existence of a link between the "pumping mode" described in the work of Berger et al..$^{5}$ and the flapping mode isolated at $S t_{D} \approx 0.10$ by Rigas et al. ${ }^{36}$ is also proposed. Both modes appear to be associated with the alternate expansion and contraction of the coherent structures forming the "hairpin vortex," with characteristic frequencies ranging from $\mathrm{St}_{\mathrm{D}}$ $=0.08$, close to the centre of the base, up to $\mathrm{St}_{\mathrm{D}}=0.095$ in proximity to the model trailing edge. The fact that the first frequency is stronger at $\mathrm{R}^{*} \approx 0$ whilst the second becomes visible only in proximity to the shear layer suggests that the peak at $\mathrm{St}_{\mathrm{D}}=0.08$ may be linked to the evolution of the transverse 
vortex forming close to the base (the "head" of the hairpin vortex), whereas the second peak may be associated with the interactions of the vortex "tails" with the vortex ring forming close to the wake closure as a result of the shear layer instability. The same interactions may also be at the origin of the third peak isolated in the pressure dataset at $\mathrm{St}_{\mathrm{D}}=0.235$. Partial confirmation to such hypothesis is given by the fact that, when higher order modes are added to the low order TPIV flow field (up to $n=10$ ), the vortex tails are occasionally seen to burst into smaller vortical structures, similar to the "vortex loops" identified in the numerical simulations performed by Mariotti et al., ${ }^{29}$ Lucas et al., ${ }^{25}$ and Evstafyeva ${ }^{12}$ and linked to the same behavior. Time resolved TPIV data, however, are needed to further confirm this model.

\section{REFERENCES}

${ }^{1}$ Alfonsi, G., "On direct numerical simulation of turbulent flows," Appl. Mech. Rev. 64(2), 020802 (2011).

${ }^{2}$ Atkinson, C. and Soria, J., "An efficient simultaneous reconstruction technique for tomographic particle image velocimetry," Exp. Fluids 47(4-5), 553 (2009).

${ }^{3}$ Auguste, F., Fabre, D., and Magnaudet, J., "Bifurcations in the wake of a thick circular disk," Theor. Comput. Fluid Dyn. 24(1), 305-313 (2010).

${ }^{4}$ Beckers, B. and Beckers, P., "A general rule for disk and hemisphere partition into equal-area cells," Comput. Geom. 45(7), 275-283 (2012).

${ }^{5}$ Berger, E., Scholz, D., and Schumm, M., "Coherent vortex structures in the wake of a sphere and a circular disk at rest and under forced vibrations," J. Fluids Struct. 4(3), 231-257 (1990).

${ }^{6}$ Bohorquez, P., Sanmiguel-Rojas, E., Sevilla, A., Jimenez-Gonzalez, J., and Martinez-Bazan, C., "Stability and dynamics of the laminar wake past a slender blunt-based axisymmetric body," J. Fluid Mech. 676, 110-144 (2011).

${ }^{7}$ Bury, Y. and Jardin, T., "Transitions to chaos in the wake of an axisymmetric bluff body," Phys. Lett. A 376(45), 3219-3222 (2012).

${ }^{8}$ Caridi, G. C. A., Ragni, D., Sciacchitano, A., and Scarano, F., "HFSB-seeding for large-scale tomographic PIV in wind tunnels," Exp. Fluids 57(12), 190 (2016).

${ }^{9}$ de la Cruz, J. M. G., Oxlade, A. R., and Morrison, J. F., "Passive control of base pressure on an axisymmetric blunt body using a perimetric slit," Phys. Rev. Fluids 2(4), 043905 (2017).

${ }^{10}$ Duell, E. G. and George, A., "Experimental study of a ground vehicle body unsteady near wake," SAE Trans. 108, 1589-1602 (1999).

${ }^{1}$ Elsinga, G. E., Scarano, F., Wieneke, B., and van Oudheusden, B. W., "Tomographic particle image velocimetry," Exp. Fluids 41(6), 933-947 (2006).

${ }^{12}$ Evstafyeva, O., "Simulation and feedback control of simplified vehicle flows," Ph.D. thesis, Imperial College London, 2018.

${ }^{13}$ Fabre, D., Auguste, F., and Magnaudet, J., "Bifurcations and symmetry breaking in the wake of axisymmetric bodies," Phys. Fluids 20(5), 051702 (2008).

${ }^{14}$ Fuchs, H. V., Mercker, E., and Michel, U., "Large-scale coherent structures in the wake of axisymmetric bodies," J. Fluid Mech. 93(1), 185-207 (1979).

${ }^{15}$ Gentile, V., Schrijer, F., Van Oudheusden, B., and Scarano, F., "Lowfrequency behavior of the turbulent axisymmetric near-wake," Phys. Fluids 28(6), 065102 (2016).

${ }^{16}$ Gentile, V., Van Oudheusden, B., Schrijer, F., and Scarano, F., "The effect of angular misalignment on low-frequency axisymmetric wake instability," J. Fluid Mech. 813, R3 (2017).

${ }^{17}$ Grandemange, M., Gohlke, M., and Cadot, O., "Bi-stability in the turbulent wake past parallelepiped bodies with various aspect ratios and wall effects," Phys. Fluids 25(9), 095103 (2013a).
${ }^{18}$ Grandemange, M., Gohlke, M., and Cadot, O., "Turbulent wake past a three-dimensional blunt body. Part 1. Global modes and bi-stability," J. Fluid Mech. 722, 51-84 (2013b).

${ }^{19}$ Grandemange, M., Gohlke, M., and Cadot, O., "Statistical axisymmetry of the turbulent sphere wake," Exp. Fluids 55(11), 1838 (2014).

${ }^{20}$ Grandemange, M., Gohlke, M., Parezanović, V., and Cadot, O., "On experimental sensitivity analysis of the turbulent wake from an axisymmetric blunt trailing edge," Phys. Fluids 24(3), 035106 (2012).

${ }^{21}$ Harris, F. J., "On the use of windows for harmonic analysis with the discrete fourier transform," Proc. IEEE 66(1), 51-83 (1978).

${ }^{22}$ Jeong, J. and Hussain, F., "On the identification of a vortex," J. Fluid Mech. 285, 69-94 (1995).

${ }^{23}$ Johl, G., Passmore, M., and Render, P., "Design methodology and performance of an indraft wind tunnel," Aeronaut. J. 108(1087), 465-473 (2004).

${ }^{24}$ Kiya, M., Ishikawa, H., and Sakamoto, H., "Near-wake instabilities and vortex structures of three-dimensional bluff bodies: A review," J. Wind Eng. Ind. Aerodyn. 89(14), 1219-1232 (2001).

${ }^{25}$ Lucas, J.-M., Cadot, O., Herbert, V., Parpais, S., and Délery, J., “A numerical investigation of the asymmetric wake mode of a squareback Ahmed bodyeffect of a base cavity," J. Fluid Mech. 831, 675-697 (2017).

${ }^{26}$ Lumley, J. L., "The structure of inhomogeneous turbulent flows," in Atmospheric Turbulence and Radio Wave Propagation, edited by A. M, Yaglom and V. I. Tatarski (Nauka, Moscow, 1967), pp. 166-178.

${ }^{27}$ Lynch, K. P. and Scarano, F., "Experimental determination of tomographic PIV accuracy by a 12-camera system," Meas. Sci. Technol. 25(8), 084003 (2014).

${ }^{28}$ Mariotti, A., "Axisymmetric bodies with fixed and free separation: Basepressure and near-wake fluctuations," J. Wind Eng. Ind. Aerodyn. 176, 21-31 (2018).

${ }^{29}$ Mariotti, A., Buresti, G., Gaggini, G., and Salvetti, M., "Separation control and drag reduction for boat-tailed axisymmetric bodies through contoured transverse grooves," J. Fluid Mech. 832, 514-549 (2017).

${ }^{30} \mathrm{Miau}, \mathrm{J} ., \mathrm{Leu}, \mathrm{T}$., Liu, T., and Chou, J., "On vortex shedding behind a circular disk," Exp. Fluids 23(3), 225-233 (1997).

${ }^{31}$ Oxlade, A. R., "High-frequency pulsed jet forcing of an axi-symmetric bluff body wake," Ph.D. thesis, Imperial College London, 2013.

${ }^{32}$ Pavia, G. and Passmore, M., "Characterisation of wake bi-stability for a square-back geometry with rotating wheels," in FKFS Conference (Springer, 2017), pp. 93-109.

${ }^{33}$ Pavia, G., Passmore, M., and Sardu, C., "Evolution of the bi-stable wake of a square-back automotive shape," Exp. Fluids 59(1), 20 (2017).

${ }^{34}$ Perry, A. K., Almond, M. T., Passmore, M., and Littlewood, R., "The study of a bi-stable wake region of a generic squareback vehicle using tomographic PIV," SAE Int. J. Passeng. Cars - Mech. Syst. 9(2), 743-753 (2016).

${ }^{35}$ Prasad, A. K., "Stereoscopic particle image velocimetry," Exp. Fluids 29(2), 103-116 (2000).

${ }^{36}$ Rigas, G., Oxlade, A., Morgans, A., and Morrison, J., "Low-dimensional dynamics of a turbulent axisymmetric wake," J. Fluid Mech. 755, R5 (2014).

${ }^{37}$ Scarano, F., "Tomographic PIV: Principles and practice," Meas. Sci. Technol. 24(1), 012001 (2012).

${ }^{38}$ Sellappan, P., McNally, J., and Alvi, F. S., "Time-averaged threedimensional flow topology in the wake of a simplified car model using volumetric PIV," Exp. Fluids 59(8), 124 (2018).

${ }^{39}$ Semeraro, O., Bellani, G., and Lundell, F., "Analysis of time-resolved PIV measurements of a confined turbulent jet using POD and Koopman modes," Exp. Fluids 53(5), 1203-1220 (2012).

${ }^{40}$ Sirovich, L., "Turbulence and the dynamics of coherent structures. Part I: Coherent structures," Q. Appl. Math. 45(3), 561-571 (1987).

${ }^{41}$ Taneda, S., "Visual observations of the flow past a sphere at Reynolds numbers between 104 and 10 6," J. Fluid Mech. 85(1), 187-192 (1978).

${ }^{42}$ Volpe, R., Devinant, P., and Kourta, A., "Experimental characterization of the unsteady natural wake of the full-scale square back Ahmed body: Flow bi-stability and spectral analysis," Exp. Fluids 56(5), 99 (2015). 
${ }^{43}$ Welch, P., "The use of fast Fourier transform for the estimation of power spectra: A method based on time averaging over short, modified periodograms," IEEE Trans. Audio Electroacoust. 15(2), 70-73 (1967).

${ }^{44}$ Westerweel, J. and Scarano, F., "Universal outlier detection for PIV data," Exp. Fluids 39(6), 1096-1100 (2005).
${ }^{45}$ Wieneke, B., "Volume self-calibration for 3D particle image velocimetry," Exp. Fluids 45(4), 549-556 (2008).

${ }^{46}$ Yang, J., Liu, M., Wu, G., Zhong, W., and Zhang, X., "Numerical study on coherent structure behind a circular disk," J. Fluids Struct. 51, 172-188 (2014). 